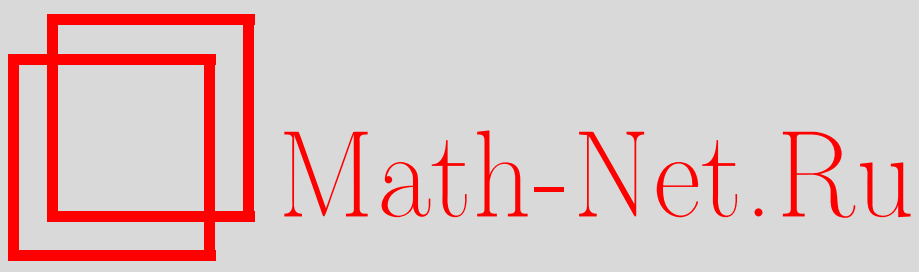

О. Н. Герман, Паруса и норменные минимумы решеток, Матем. сб., 2005, том 196, номер 3, 31-60

DOI: https://doi.org/10.4213/sm1272

Использование Общероссийского математического портала Math-Net.Ru подразумевает, что вы прочитали и согласны с пользовательским соглашением

http: //www. mathnet.ru/rus/agreement

Параметры загрузки:

IP : 54.224 .60 .19

26 апреля 2023 г., $14: 18: 05$ 
УДК 511.9

\title{
О.Н. Герман
}

\section{Паруса и норменные минимумы решеток}

\begin{abstract}
Известно, что число плохо приближаемо в том и только том случае, если его неполные частные равномерно ограничены. В работе доказано аналогичное утверждение для так назьваемых парусов, одного из наиболее естественных многомерных обобщений цепных дробей.

Библиографйя: 19 названий.
\end{abstract}

\section{§1. Введение}

Число $\alpha$ называется плохо приближаемым, если существует такая константа $c>0$, что для всех целых $p$ и натуральных $q$ выполняется соотношение

$$
|q \alpha-p| \geqslant \frac{c}{q}
$$

Общеизвестно следуюшее

УТВЕРЖДЕНИЕ 1. Иррачиональное число плохо приближаемо в том и только том случае, если его неполные частные равномерно ограниченьи.

Можно дать следующую геометрическую интерпретацию этого факта. Рассмотрим две прямые в $\mathbb{R}^{2}$, порожденные векторами $(1, \alpha)$ и $(1, \beta), \alpha, \beta \in \mathbb{R} \backslash \mathbb{Q}$, $\alpha>0>\beta>-1$. Эти прямые разбивают плоскость на четыре угла (которые мы будем называть конусами согласно терминологии будуших многомерных обобщений). Во внутренности каждого конуса рассмотрим выпуклую оболочку целочисленных точек. Получившиеся четыре неограниченных выпуклых многоугольника называются полигонами Клейна. Имеет место замечательное соответствие (см. [1]) между вершинами полигонов Клейна и подходящими дробями чисел $\alpha$ и $\beta$, при котором вершине $(q, p)$, если $q>0$, соответствует подходящая дробь $p / q$ числа $\alpha$ или $\beta$, и наоборот. Аналогичное соответствие имеется между неполными частными чисел $\alpha$ и $\beta$ и целочисленными длинами ребер полигонов Клейна. Используя эти соответствия, получаем, что, с одной стороны, числа $\alpha$ и $\beta$ имеют равномерно ограниченные неполные частные в том и только том случае, если целочисленные длины ребер соответствующих полигонов Клейна равномерно ограничены. С другой стороны, числа $\alpha$ и $\beta$ оба являются плохо приближаемыми тогда и только тогда, когда inf $|(q \alpha-p)(q \beta-p)|>0$, где inf берется по всем целым $p$ и $q$, не равньм одновременно нулю. Если теперь положить

$$
\varphi_{\alpha, \beta}\left(x_{1}, x_{2}\right)=\left(x_{1} \alpha-x_{2}\right)\left(x_{1} \beta-x_{2}\right),
$$

то утверждение 1 можно переформулировать так:

Работа вьполнена при поддержке Российского фонда фундаментальных исследований (грант № 02-01-00912), фонда INTAS (грант № 03-51-5070) и Программы поддержки ведущих научных школ РФ (грант № НШ-136.2003.1).

(C) О.Н. Герман 2005 
УТВЕРЖДЕНИЕ 2. Если заданы произвольные различные иррациональные числа $\alpha$ и $\beta$, то челочисленные длины ребер полигонов Клейна, построенных описанным выше способом, равномерно ограничены тогда и только тогда, $\kappa о 2 \partial a$

$$
\inf _{\mathbf{x} \in \mathbb{Z}^{2} \backslash\{\mathbf{0}\}}\left|\varphi_{\alpha, \beta}(\mathbf{x})\right|>0
$$

Оказывается, имеет место многомерный аналог этого утверждения, доказательство которого и является целью настоящей работы.

Если в двумерном случае главную роль играют границы полигонов Клейна, являюшиеся, по сути, геометрической интерпретацией понятия цепных дробей, то в произвольной размерности основными объектами исследования будут так называемые паруса, границы полиэдров Клейна (точное определение будет дано в $\$ 2$ ). Это одно из наиболее изящных многомерных обобщений цепных дробей, в последнее время пользующееся все большей популярностью. Наиболее изученными являются паруса, соответствующие алгебраическим конусам (см. [2]-[10]). О некоторых свойствах произвольных парусов см. работы [11] и [12]. Стоит также отметить работу [13], в которой получен ряд статистических результатов о парусах.

\section{§2. Основные понятия и формулировки результатов}

На протяжении всей работы мы будем через $\rho(\cdot, \cdot)$ обозначать евклидово расстояние, через $\operatorname{conv}(F), \operatorname{aff}(F)$ и $\operatorname{vol}_{k} F-$ соответственно выпуклую оболочку, аффинную оболочку и $k$-мерный объем $k$-мерного множества $F$. Плоскость $\pi$ будем называть вполне рациональной относительно решетки $\Lambda$, если $\operatorname{dim}(\Lambda \cap \pi)=\operatorname{dim} \pi$. Стандартный ортонормированный базис $\mathbb{R}^{n}$ будем обозначать $\mathscr{E}=\left\{\mathbf{e}_{1}, \ldots, \mathbf{e}_{n}\right\}$.

Рассмотрим произвольную $n$-мерную решетку $\Lambda \subset \mathbb{R}^{n}$ и произвольный невырож денный симплициальный конус $\mathscr{C} \subset \mathbb{R}^{n}$ с ребрами, порожденньми векторами $\boldsymbol{\omega}_{1}, \ldots, \boldsymbol{\omega}_{n}$ :

$$
\mathscr{C}=\left\{\lambda_{1} \omega_{1}+\cdots+\lambda_{n} \omega_{n} \mid \lambda_{i} \geqslant 0\right\}
$$

ОПРЕДЕЛЕНИЕ 1. Множество $K=\operatorname{conv}(\mathscr{C} \cap \Lambda \backslash\{\boldsymbol{0}\})$ называется полиэдром Клейна.

В настоящей работе мы ограничимся случаем, когда решетка $\Lambda$ иррациональна относительно конуса $\mathscr{C}$, т.е. когда в плоскостях гиперграней $\mathscr{C}$ нет точек решетки, кроме 0. Тогда, как показано в [14], полиэдр Клейна является обобшенным многогранником, т.е. его пересечение с любым ограниченным многогранником тоже будет многогранником. Стало быть, граница $K$ в этом случае является $(n-1)$-мерной полиэдральной поверхностью, гомеоморфной $\mathbb{R}^{n-1}$ и состоящей из вьпуклых $(n-1)$-мерных (обобщенных) многогранников, среди которых, вообще говоря, могут встретиться неограниченные. Но в любом случае каждая точка, лежащая на гранище $K$, принадлежит лишп конечному числу граней $K$.

ОПРЕДЕЛЕНИЕ 2. Граница П полиэдра Клейна $K$ называется парусом.

Грани паруса размерности $n-1$ будут играть роль неполных частных. В качестве численной характеристики этих “неполных частных" мы будем рассматривать их "определители". 
ОпРЕДЕЛЕНИЕ 3 . Если $F \subset \mathbb{R}^{n}$-произвольный $(n-1)$-мерный многогранник, то под определителем $F$ мы будем понимать величину

$$
\operatorname{det} F=\operatorname{vol}_{n}(\operatorname{conv}(F \cup\{\mathbf{0}\})) \cdot n ! .
$$

Очевидно, что если $F$ - симплекс, то $\operatorname{det} F$ совпадает с определителем матрицп, составленной из координат вершин $F$. Следовательно, при $n=2$, когда парус одномерен, определители ребер паруса равны целочисленным длинам этих ребер.

В дальнейшем важную роль будут играть сечения конуса $\mathscr{C}$, содержащие $(n-1)$ мерные грани П. Если $F$ - такая грань, то положим

$$
S(F)=\operatorname{aff}(F) \cap \mathscr{C} .
$$

Векторы $\boldsymbol{\omega}_{1}, \ldots, \boldsymbol{\omega}_{n}$, порождающие ребра $\mathscr{C}$, образуют базис $\mathbb{R}^{n}$. Каждый из $2^{n}$ наборов $\left\{ \pm \boldsymbol{\omega}_{1}, \ldots, \pm \boldsymbol{\omega}_{n}\right\}$ также является базисом $\mathbb{R}^{n}$ и задает соответствуюший конус, в котором можно взять выпуклую оболочку точек решетки $\Lambda$ и получить парус. Таким образом, решетка $\Lambda$ и конус $\mathscr{C}$ порождают $2^{n}$ парусов.

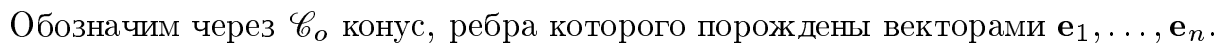
До сих пор мы рассматривали парус, соответствуюший произвольному симплициальному конусу $\mathscr{C}$ и произвольной решетке $\Lambda$, иррациональной относительно $\mathscr{C}$. Однако очевидно, что любой парус по модулю действия группы $\mathrm{GL}(n, \mathbb{R})$ совпадает с парусом, соответствуюшим конусу $\mathscr{C}_{o}$ и некоторой иррациональной относительно него решетке (такую решетку мы будем называть иррациональной, опуская слова “относительно $\mathscr{C}_{o}$ ”). С другой стороны, можно зафиксировать решетку $\mathbb{Z}^{n}$ и брать произвольный конус, относительно которого $\mathbb{Z}^{n}$ иррациональна (в этом случае будем называть иррациональным конус). Мы будем использовать обе эти точки зрения.

Положим

$$
\varphi_{o}(\mathbf{x})=x_{1} \cdots x_{n}
$$

и будем говорить, что форма $\varphi(\mathbf{x})$ соответствует конусу $\mathscr{C}$, если

$$
\varphi(\mathbf{x})=\varphi_{o}\left(W^{-1} \mathbf{x}\right) \operatorname{det} W,
$$

где $W$ - матрица, составленная из координат векторов $\boldsymbol{\omega}_{1}, \ldots, \boldsymbol{\omega}_{n}$ (в $i$-м столбцекоординаты $\left.\boldsymbol{\omega}_{i}\right)$. Форма $\varphi_{o}$, очевидно, соответствует конусу $\mathscr{C}_{o}$.

ОПРЕДЕЛЕНИЕ 4. Норменнбцм минимумом решетки $\Lambda$ называется величина

$$
N(\Lambda)=\inf _{\mathbf{x} \in \Lambda \backslash\{0\}}\left|\varphi_{o}(\mathbf{x})\right| .
$$

Теперь, когда даны все основные определения, мы можем сформулировать основной результат настоящей работы.

Теорема 1. Норменный минимум п-мерной иррациональной решетки $\Lambda \subset \mathbb{R}^{n}$ отличен от нуля тогда и только тогда, когда определители граней каждого из $2^{n}$ парусов, порожденных решеткой $\Lambda$ и конусом $\mathscr{C}_{\circ}$, равномерно ограничень. 
Теорема 1 непосредственно вытекает из следующих двух теорем.

Теорема 2. Норменный минимум $п$-мерной иррациональной решетки $\Lambda \subset \mathbb{R}^{n}$ отличен от нуля тогда и только тогда, когда существует такая константа $D$, что для каждой грани $F$ каждого из $2^{n}$ парусов, порожденных решеткой $\Lambda, \operatorname{det} S(F)<D$.

При этом $D$ будет зависеть лишь от $N(\Lambda)$ и от $n$. И наоборот, если задана константа $D$, то найдется константа $\mu>0$, зависящая только от $D$ u от $n$, такая, что $N(\Lambda)>\mu$.

ТеОРема 3. Пусть задана п-мерная иррациональная решетка $\Lambda \subset \mathbb{R}^{n}$. Пусть П - парус, соответствующий решетке $\Lambda$ и конусу $\mathscr{C}_{o}$. Пусть определители граней паруса равномерно ограничены константой $D$. Тогда все определители $\operatorname{det} S(F)$, где $S(F)$ - сечение конуса $\mathscr{C}_{о}$, содержащее грань $F$ паруса П, также равномерно ограничены константой $D^{\prime}$, зависящей лишь oт $D$ u om $n$.

ЗАмечАниЕ. В формулировке теоремы 1 ничего не сказано о том, как зависят друг от друга норменный минимум решетки и максимум определителей граней парусов. Однако, как легко видеть из теорем 2 и 3 , при заданном значении $N(\Lambda)$ существует эффективная оценка сверху на определители граней парусов. И наоборот, при заданном значении максимума определителей граней парусов существует эффективная оценка снизу на $N(\Lambda)$.

Теорему 3 мы докажем сначала в трехмерном случае (в $\S 6$ ). При $n=3$ доказательство весьма прозрачно, в то время как при $n \geqslant 4$ возникают определенные технические трудности, в связи с чем доказательство становится достаточно громоздким. Доказательство теоремы 3 для произвольного $n$ дано в $\S 7$.

Отметим также, что мы не стремимся получить точный вид констант. Поэтому мы порой жертвуем точностью оценок ради их компактности.

\section{§ 3. Связь с проблемами Литтлвуда и Оппенгейма}

Следующие две гипотезы являются классическими.

ГиПотеЗА ЛиттЛвУДА. Если $\alpha, \beta \in \mathbb{R}, m o \inf _{m \in \mathbb{N}} m\|m \alpha\|\|m \beta\|=0$.

ГИПОТЕЗА ОППЕНГЕЙМА. Если $n \geqslant 3 u \Lambda \subset \mathbb{R}^{n}-n$-мерная решетка такая, что $N(\Lambda)>0$, то $\Lambda$ - алгебраическая решетка (т.е. подобна решетке полного модуля чисто вещественного алгебрачческого поля степени $n$ ).

Известно (см. [15]), что из трехмерной гипотезы Оппенгейма следует гипотеза Литтлвуда. В работах [16] и [17] была предпринята попытка доказать гипотезу Оппенгейма, однако в доказательстве имеется весьма существенный пробел. По этой причине обе гипотезы остаются недоказанньми.

Основной результат настоящей статьи позволяет переформулировать гипотезу Оппенгейма следующим образом. В силу теоремы 1 положительность норменного минимума решетки $\Lambda$ равносильна равномерной ограниченности определителей граней парусов, порожденных $\Lambda$ и конусом $\mathscr{C}_{o}$. Далее, алгебраичность решетки $\Lambda$ равносильна сушествованию свободной абелевой группы ранга $n-1$ автоморфизмов $\Lambda$, сохраняющих паруса (в одну сторону - это прямое следствие теоремы Дирихле о единицах, см., например, [18], а в другую - см. [5] или [6]). Таким образом, 
в алгебраическом случае мы можем говорить об $(n-1)$-периодичности парусов (в смысле существования $(n-1)$-параметрической группы “периодов"; при этом фундаментальные области при действии этой групшы компактны и, стало быть, состоят из конечного числа граней).

Суммируя сказанное, получаем переформулировку гипотезы Оппенгейма в терминах свойств парусов:

ПЕРЕФОРМУЛИРОВКА ГИПОТЕЗЫ ОППЕНГЕЙМА. Если $n \geqslant 3$, то равномерная ограниченность определителей граней всех парусов, порожсденных $n$-мерной решеткой $\Lambda \subset \mathbb{R}^{n}$ и конусом $\mathscr{C}_{\text {o }}$, влечет $(n-1)$-периодичность этих парусов.

Общеизвестно, что в случае $n=2$ утверждение гипотезы неверно, так как числа с ограниченными неполными частными не исчерпьваются квадратичными иррациональностями.

\section{§4. Двойственный конус}

Пусть ребра конуса $\mathscr{C}$, как и прежде, порождены векторами $\boldsymbol{\omega}_{1}, \ldots, \boldsymbol{\omega}_{n}$, а $W$ - матрица, составленная из координат этих векторов. Рассмотрим векторы $\boldsymbol{\omega}_{1}^{*}, \ldots, \boldsymbol{\omega}_{n}^{*}$ такие, что $\left(\boldsymbol{\omega}_{i}, \boldsymbol{\omega}_{j}^{*}\right)=\delta_{i j}$. Эти векторы задают конус $\mathscr{C}^{*}$, двойственньй конусу $\mathscr{C}$, т.е.

$$
\mathscr{C}^{*}=\left\{\mathbf{x} \in \mathbb{R}^{n} \mid \forall \mathbf{y} \in \mathscr{C} \quad(\mathbf{x}, \mathbf{y}) \geqslant 0\right\}
$$

Лемма 1. Пусть конус $\mathscr{C}$ иррационален. Тогда следующие два утверәсдения әквивалентны:

1) конус $\mathscr{C}^{*}$ иррачионален;

2) любая грань любого из $2^{n}$ парусов, порождаемых решеткой $\mathbb{Z}^{n}$ иконусом $\mathscr{C}$, ограничена.

ДокАЗАТЕЛьство. 1) $\Rightarrow 2$ ). Если у какого-то из парусов, порож даемых решеткой $Z^{n}$ и конусом $\mathscr{C}$, есть неограниченная грань, то она обязана быть параллельна какому-то ребру $\ell$ конуса $\mathscr{C}$. Это значит, что через $\ell$ проходит вполне рациональная гиперплоскость $\pi$, нормаль $\mathbf{n}_{\pi}$ к которой лежит в плоскости одной из граней конуса $\mathscr{C}^{*}$. Но $\exists \lambda>0: \lambda \mathbf{n}_{\pi} \in \mathbb{Z}^{n}$, стало быть, $\mathscr{C}^{*}$ не иррационален.

$2) \Rightarrow 1)$. Пусть в плоскости какой-то грани конуса $\mathscr{C}^{*}$ есть целая точка. Тогда через соответствующее ребро $\ell$ конуса $\mathscr{C}$ проходит некоторая вполне рациональная гиперплоскость $\pi$. Но тогда среди $2^{n-1}$ конусов, содержащих $\ell$, найдется такой, парус которого имеет неограниченную грань, параллельную $\pi$.

ОПРЕДЕЛЕНИЕ 5. Пусть $A$-произвольное подмножество $\mathbb{R}^{n}$. Тогда полярным множеством для множества $A$ будем назьвать множество

$$
A^{\circ}=\left\{\mathbf{x} \in \mathbb{R}^{n} \mid \forall \mathbf{y} \in A \quad(\mathbf{x}, \mathbf{y}) \geqslant 1\right\}
$$

Рассмотрим полиэдр Клейна $K$, соответствующий конусу $\mathscr{C}$ (как и прежде, иррациональному). Для каждой $(n-1)$-мерной грани $F$ полиэдра $K$ обозначим че- 
рез $\mathbf{n}_{F}$ нормаль к плоскости $\operatorname{aff}(F)$, направленную в полупространство, содержащее $K$ (тогда $\left(\mathbf{x}, \mathbf{n}_{F}\right)=1$ для всех $\left.\mathbf{x} \in \mathrm{aff}(F)\right)$. Положим

$$
\begin{aligned}
H_{F} & =\left\{\mathbf{x} \in \mathbb{R}^{n} \mid\left(\mathbf{x}, \mathbf{n}_{F}\right) \geqslant 1\right\}, \\
\ell_{F} & =\left(H_{F}\right)^{\circ}=\left\{\lambda \mathbf{n}_{F} \mid \lambda \geqslant 1\right\}
\end{aligned}
$$

и

$$
K^{\prime}=\overline{\operatorname{conv}\left(\bigcup_{F} \ell_{F}\right)}
$$

где объединение берется по всем граням $K$, а черта означает замькание.

Лемма 2. $K^{\circ}=K^{\prime}$.

ДокаЗАТЕЛЬСтво. Пусть $\mathbf{x} \in \operatorname{conv}\left(\bigcup_{F} \ell_{F}\right)$. Тогда по теореме Каратеодори (см., например, [19]) сушествуют $n+1$ граней $F_{1}, \ldots, F_{n+1}$ таких, что $\mathbf{x} \in$ $\operatorname{conv}\left(\ell_{F_{1}} \cup \cdots \cup \ell_{F_{n+1}}\right)$. То есть $\mathbf{x}=\lambda_{1} \mathbf{n}_{F_{1}}+\cdots+\lambda_{n+1} \mathbf{n}_{F_{n+1}}$, где $\lambda_{i} \geqslant 0$ и $\lambda_{1}+\cdots+\lambda_{n+1} \geqslant 1$. Отсюда, замечая, что $\left(\mathbf{y}, \mathbf{n}_{F}\right) \geqslant 1$ для любой грани $F$ и любой точки $\mathbf{y} \in K$, получаем, что $(\mathbf{x}, \mathbf{y}) \geqslant 1$ для всех $\mathbf{y} \in K$. Стало быть, мы доказали, что выпуклая оболочка $\operatorname{conv}\left(\bigcup_{F} \ell_{F}\right)$ содержится в $K^{\circ}$. Но линейные функционалы на $\mathbb{R}^{n}$ непрерьвны, следовательно, и замыкание этой выпуклой оболочки также содержится в $K^{\circ}$.

Пусть теперь $\mathbf{x} \notin K^{\prime}$. Рассмотрим отрезок $[\mathbf{0}, \mathbf{x}]$. Этот отрезок и множество $K^{\prime}$ не имеют общих точек, поскольку вместе с каждой своей точкой а множество $K^{\prime}$ содержит луч $\{\lambda \mathbf{a} \mid \lambda \geqslant 1\}$. Следовательно, в силу выпуклости и замкнутости $K^{\prime}$ отрезок $[\mathbf{0}, \mathbf{x}]$ строго отделим от $K^{\prime}$, т.е. найдется такой линейный функционал $L_{\mathbf{y}}(\cdot)=(\mathbf{y}, \cdot)$, что

$$
L_{\mathbf{y}}(\mathbf{z})>1>L_{\mathbf{y}}(\mathbf{x})
$$

для любой точки $\mathbf{z} \in \bigcup_{F} \ell_{F}$. Первое из этих неравенств дает нам, что

$$
\mathbf{y} \in \bigcap_{F}\left(\ell_{F}\right)^{\circ}=\bigcap_{F} H_{F}=K .
$$

Следовательно, из второго неравенства в (2) получаем, что $\mathbf{x} \notin K^{\circ}$.

Предположим теперь, что конус $\mathscr{C}^{*}$ иррационален. Тогда в $\mathscr{C}^{*}$ можно определить полиэдр Клейна (относительно решетки $\mathbb{Z}^{n}$ ). Обозначим его $K^{*}$.

Из (1) видим, что $(\mathbf{x}, \mathbf{y}) \geqslant 0$ для всех $\mathbf{x} \in \mathscr{C}^{*}, \mathbf{y} \in \mathscr{C}$, причем равенство может достигаться только на границах конусов. Следовательно, если $\mathbf{x} \in K^{*}, \mathbf{y} \in K$, то $(\mathbf{x}, \mathbf{y})>0$. Если же $\mathbf{x} \in K^{*} \cap \mathbb{Z}^{n}, \mathbf{y} \in K \cap \mathbb{Z}^{n}$, то $(\mathbf{x}, \mathbf{y}) \geqslant 1$. Вспоминая, что $K$ и $K^{*}$ суть выпуклые оболочки целых точек, получаем, что $(\mathbf{x}, \mathbf{y}) \geqslant 1$ для всех $\mathbf{x} \in K^{*}, \mathbf{y} \in K$. Стало быть,

$$
K^{*} \subseteq K^{\circ} \text { и } K \subseteq\left(K^{*}\right)^{\circ} .
$$

Рассмотрим произвольную $(n-1)$-мерную грань $F$ полиэдра $K$ и соответствуюшую ей точку $\mathbf{n}_{F}$. Тогда имеет место следуюший факт: 
Лемма 3. Пусть $\varphi^{*}-$ форма, соответствующая конусу $\mathscr{C}^{*}$. Тогда

$$
\varphi^{*}\left(\mathbf{n}_{F}\right) \operatorname{det} S(F)=1
$$

ДокАЗАТЕльство. Вершинами симплекса $S(F)$ являются точки $\lambda_{1} \omega_{1}, \ldots$, $\lambda_{n} \boldsymbol{\omega}_{n}$, где $\lambda_{1}, \ldots, \lambda_{n}$ - некоторые положительные числа. Значит, $\operatorname{det} S(F)=$ $\operatorname{det}\left(\lambda_{1} \boldsymbol{\omega}_{1}, \ldots, \lambda_{n} \boldsymbol{\omega}_{n}\right)$. С другой стороны, $\exists \mu_{1}, \ldots, \mu_{n}>0: \mathbf{n}_{F}=\mu_{1} \boldsymbol{\omega}_{1}^{*}+\cdots+$ $\mu_{n} \boldsymbol{\omega}_{n}^{*}$. Отсюда и непосредственно из определения функции $\varphi^{*}$ следует, что

$$
\varphi^{*}\left(\mathbf{n}_{F}\right)=\operatorname{det}\left(\mu_{1} \mathbf{e}_{1}, \ldots, \mu_{n} \mathbf{e}_{n}\right) \operatorname{det}\left(\omega_{1}^{*}, \ldots, \omega_{n}^{*}\right)=\operatorname{det}\left(\mu_{1} \omega_{1}^{*}, \ldots, \mu_{n} \omega_{n}^{*}\right)
$$

Отметим также, что $\lambda_{i} \mu_{i}=1$, поскольку $\left(\mathbf{n}_{F}, \lambda_{i} \boldsymbol{\omega}_{i}\right)=1$.

Следовательно,

$$
\begin{aligned}
\varphi^{*}\left(\mathbf{n}_{F}\right) \operatorname{det} S(F) & =\operatorname{det}\left(\mu_{1} \boldsymbol{\omega}_{1}^{*}, \ldots, \mu_{n} \boldsymbol{\omega}_{n}^{*}\right) \operatorname{det}\left(\lambda_{1} \boldsymbol{\omega}_{1}, \ldots, \lambda_{n} \boldsymbol{\omega}_{n}\right) \\
& =\operatorname{det}\left(\boldsymbol{\omega}_{1}^{*}, \ldots, \boldsymbol{\omega}_{n}^{*}\right) \operatorname{det}\left(\boldsymbol{\omega}_{1}, \ldots, \boldsymbol{\omega}_{n}\right)=\operatorname{det}\left(W^{T} W^{*}\right)=\operatorname{det}(E)=1 .
\end{aligned}
$$

СлеДСтвиЕ. Если $\operatorname{det} S(F) \leqslant D$ для каждой грани $F$ каждого из $2^{n}$ парусов, порожденных решеткой $\mathbb{Z}^{n}$ и конусом $\mathscr{C}$, то

$$
\forall \mathbf{x} \in \mathbb{Z}^{n} \backslash\{0\} \quad\left|\varphi^{*}(\mathbf{x})\right| \geqslant D^{-1}
$$

ДоказАТЕльство. Докажем, что $\varphi^{*}(\mathbf{x}) \geqslant D^{-1}$ для любого $\mathbf{x} \in \mathscr{C}^{*} \cap \mathbb{Z}^{n} \backslash\{\mathbf{0}\}$. Для остальных конусов, порождаемых конусом $\mathscr{C}^{*}$, доказательство аналогично.

Из леммы 3 следует, что $\varphi^{*}\left(\mathbf{n}_{F}\right) \geqslant D^{-1}$ для любой грани $F$ полиэдра $K$. Применяя лемму 2 , получаем, что $\varphi^{*}(\mathbf{x}) \geqslant D^{-1}$ для всех $\mathbf{x} \in K^{\circ}$ (чтобы в этом убедиться, достаточно показать, что если $\varphi_{o}(\mathbf{x})=\varphi_{o}(\mathbf{y})=1$, то $\varphi_{o}((\mathbf{x}+\mathbf{y}) / 2) \geqslant 1, \mathrm{a}$ это сразу следует из неравенства между средним арифметическим и средним геометрическим). Далее, по лемме 1 из ограниченности граней парусов, порожденных решеткой $\mathbb{Z}^{n}$ и конусом $\mathscr{C}$, следует иррациональность конуса $\mathscr{C}^{*}$. Следовательно, мы можем воспользоваться первым соотношением из (3) и получить, что $\mathscr{C}^{*} \cap \mathbb{Z}^{n} \backslash\{\mathbf{0}\} \subset K^{*} \subseteq K^{\circ}$. Значит, для всех $\mathbf{x} \in K^{*}$ также имеет место неравенство $\varphi^{*}(\mathbf{x}) \geqslant D^{-1}$.

\section{§5. Доказательство теоремы 2}

В одну сторону теорема 2 была известна еще Скубенко. А именно имеет место следуюшее утверждение.

Лемма 4. Если $N(\Lambda) \geqslant \mu>0$, то существует константа $D$, зависящая только от $\mu$ и от п, такая, что для каждой грани F каждого из $2^{n}$ парусов, порожсднных решеткой $\Lambda$, $\operatorname{det} S(F)<D$.

Для полноты изложения мы приводим доказательство этой леммы, которое следует рассуждениям Скубенко из [17]. 
ДокАЗАТЕЛЬСТво. Рассмотрим произвольную грань $F$ одного из $2^{n}$ парусов, порож денных решеткой $\Lambda$. Не ограничивая общности, будем считать, что этот парус содержится в конусе $\mathscr{C}_{o}$. Применив подходящий гиперболический поворот, мы можем считать, что $F$ перпендикулярно биссектрисе конуса $\mathscr{C}_{o}$. (Под гиперболическим поворотом мы понимаем линейное преобразование, задаюшееся диагональной матрицей с определителем 1 и положительными элементами на диагонали; очевидно, такое преобразование не меняет ни комбинаторной структуры паруса, ни определителей его граней.)

Положим $\mathbf{v}=(1, \ldots, 1)$. Рассмотрим в плоскости $(\mathbf{x}, \mathbf{v})=0$ открытый $(n-1)$ мерньй шар $B_{\mu}$ радиуса $\frac{1}{2} \mu^{1 / n}$ с центром в $\mathbf{0}$. Наконец, рассмотрим (открытый) цилиндр

$$
\Omega=\left\{\mathbf{x}+\lambda \mathbf{v}\left|\mathbf{x} \in B_{\mu},\right| \lambda \mid<\rho(\mathbf{0}, S(F))\right\} .
$$

Докажем, что для любой точки $\mathbf{x}$ из множества $\Omega \backslash\left( \pm \mathscr{C}_{o}\right)$ выполняется неравенство $\left|x_{1} \cdots x_{n}\right|<\mu$. Пусть $\mathbf{x}-$ такая точка, и пусть $\mathscr{C}_{o}^{\prime}-$ конус, смежньй (хотя бы по ребру) с конусом $\mathscr{C}_{o}$, содержаший точку х. Тогда существуют такие $\mathbf{y} \in B_{\mu} \cap \mathscr{C}_{o}^{\prime}$ и $\lambda \in \mathbb{R}$, что $\mathbf{x}=\mathbf{y}+\lambda \mathbf{v}$. Поскольку $\mathbf{y} \in B_{\mu}$, то $\max \left(\left|y_{1}\right|, \ldots,\left|y_{n}\right|\right)<\frac{1}{2} \mu^{1 / n}$. Но соответствующие координаты точек $\mathbf{x}$ и $\mathbf{y}$ не могут иметь разные знаки. Следовательно, $|\lambda| \leqslant \max \left(\left|y_{1}\right|, \ldots,\left|y_{n}\right|\right)<\frac{1}{2} \mu^{1 / n}$, откуда получаем, что $\max \left(\left|x_{1}\right|, \ldots,\left|x_{n}\right|\right)<\mu^{1 / n}$. Стало быть, $\left|x_{1} \cdots x_{n}\right|<\mu$.

Отсюда, вспоминая, что $N(\Lambda) \geqslant \mu$, видим, что множество $\Omega \backslash\left( \pm \mathscr{C}_{o}\right)$ не содержит ни одной точки решетки $\Lambda$. С другой стороны, множество $\Omega \cap \mathscr{C}_{o} \backslash\{\boldsymbol{0}\}$ также не содержит точек $\Lambda$, поскольку $F$ - грань паруса. Стало быть, $\Omega \cap \Lambda=\{\boldsymbol{0}\}$. Из теоремы Минковского о выпуклом теле получаем, что

$$
\operatorname{vol}_{n} \Omega \leqslant 2^{n} .
$$

Следовательно,

т.e.

$$
\rho(\mathbf{0}, S(F)) \leqslant \frac{2^{n-1}}{\operatorname{vol}_{n-1} B_{\mu}}<\frac{(n-1) !}{\mu^{n-1}},
$$

$$
\operatorname{det} S(F)=(\sqrt{n} \rho(\mathbf{0}, S(F)))^{n}<\left(\frac{\sqrt{n}(n-1) !}{\mu^{n-1}}\right)^{n}<\left(\frac{n}{\mu}\right)^{n^{2}} .
$$

Остается положить

$$
D=\left(\frac{n}{\mu}\right)^{n^{2}} .
$$

ЗАмЕчАниЕ. В изложенном доказательстве мы пользовались тем фактом, что подходящим гиперболическим поворотом можно добиться того, чтобы грань $F$ стала перпендикулярна биссектрисе конуса $\mathscr{C}$. Но это возможно в том и только том случае, если $F$ ограничена. Если же $F$ не ограничена, то гиперболическим поворотом мы можем добиться только того, чтобы нормаль к плоскости $\operatorname{aff}(F)$ стала сколь угодно близка к биссектрисе $\mathscr{C}$. Однако, с другой стороны, при таком повороте расстояние от $\mathbf{0}$ до $\mathrm{aff}(F)$ можно сделать сколь угодно большим. Тогда объем цилиндра $\Omega$ будет сколь угодно велик, чего не может быть в силу теоремы Минковского. Стало быть, в предположении леммы все грани всех $2^{n}$ парусов должны быть ограничены.

Теперь нам нужно доказать обратное утверждение. 
Лемма 5. Если существует константа $D$ такая, что для каждой грани $F$ каждого из $2^{n}$ парусов, порожденных решеткой $\Lambda$, $\operatorname{det} S(F) \leqslant D$, то найдется такое $\mu>0$, зависящее только от $D$ и от $n$, что $N(\Lambda) \geqslant \mu$.

Далее в этом параграфе нам будет удобно иметь дело с “двойственными” формулировками. Рассмотрим оператор $A \in \mathrm{GL}(n, \mathbb{R}): A \Lambda=\mathbb{Z}^{n}$. Тогда $A$ переводит $\mathscr{C}_{o}$ в некоторый конус $\mathscr{C}$, которому соответствует форма $\varphi$, и леммы 4,5 можно переформулировать следуюшим образом.

Лемма $4^{\prime}$. Если

$$
\inf _{\mathbf{x} \in \mathbb{Z}^{n} \backslash\{\mathbf{0}\}}|\varphi(\mathbf{x})| \geqslant \mu>0,
$$

то найдется константа $D$, зависящая только от $\mu$ и от $n$, такая, что если $F$ - произвольная грань любого из $2^{n}$ парусов, порождаемых решеткой $Z^{n}$ и конусом $\mathscr{C}$, mо $\operatorname{det} S(F) \leqslant D$.

Лемма 5'. Если существует константа $D$ такая, что для каждой грани $F$ каждого из $2^{n}$ парусов, порожденных решеткой $\mathbb{Z}^{n}$ и конусом $\mathscr{C}$, $\operatorname{det} S(F) \leqslant D$, то найдется такое $\mu>0$, зависящее только от $D$ и от $n$, чmo

$$
\inf _{\mathbf{x} \in \mathbb{Z}^{n} \backslash\{\mathbf{0}\}}|\varphi(\mathbf{x})| \geqslant \mu .
$$

Лемма 5' вытекает из леммы 3.

ДоКАЗАТЕЛЬСТво ЛЕммЫ 5'. Воспользуемся следствием леммы 3 и получим, что

$$
\forall \mathbf{x} \in \mathbb{Z}^{n} \backslash\{\mathbf{0}\} \quad\left|\varphi^{*}(\mathbf{x})\right| \geqslant D^{-1},
$$

где $\varphi^{*}$ - форма, соответствуюшая конусу $\mathscr{C}^{*}$. Но тогда по лемме $4^{\prime}$, примененной к конусу $\mathscr{C}^{*}$, найдется константа $D^{\prime}$, зависящая только от $D$ и от $n$, такая, что $\operatorname{det} S(F) \leqslant D^{\prime}$ для всякой грани $F$ любого из $2^{n}$ парусов, порождаемых решеткой $Z^{n}$ и конусом $\mathscr{C}^{*}$.

Применим опять следствие леммы 3 , на этот раз к конусу $\mathscr{C}^{*}$, и получим, что

$$
\forall \mathbf{x} \in \mathbb{Z}^{n} \backslash\{\mathbf{0}\} \quad|\varphi(\mathbf{x})| \geqslant\left(D^{\prime}\right)^{-1}
$$

Остается положить $\mu=\left(D^{\prime}\right)^{-1}$.

Стоит отметить, что в [17] было доказано, что норменные минимумы двойственных решеток одновременно отличны от нуля. Из этого факта и из следствия леммы 3 можно сразу получить, что в предположении леммы $5^{\prime}$ для некоторого $\mu>0$ вьполняется соотношение

$$
\inf _{\mathbf{x} \in \mathbb{Z}^{n} \backslash\{\mathbf{0}\}}|\varphi(\mathbf{x})| \geqslant \mu .
$$

Однако лемма $5^{\prime}$ утверждает большее, а именно, что $\mu$ можно выбрать так, чтобы оно зависело лишш от $D$ и от $n$. 


\section{§6. Доказательство теоремы 3 в случае $n=3$}

Итак, мы предполагаем, что определители граней паруса П равномерно ограничены константой $D$.

Положим

$$
D^{\prime}=12 D(D+2)^{2}
$$

и допустим, что сушествует такая грань $F$ паруса П, что

$$
\operatorname{det} S(F) \geqslant D^{\prime} \text {. }
$$

Применив подходящий гиперболический поворот (который, очевидно, не изменит ни комбинаторной структуры паруса, ни определителей его граней), мы можем, не ограничивая обшности, считать, что $F$ перпендикулярно биссектрисе конуса $\mathscr{C}_{o}$.

Обозначим вершины $S(F)$ через $\mathbf{v}_{1}, \mathbf{v}_{2}, \mathbf{v}_{3}$. Из (5) следует, что

$$
\left|\mathbf{v}_{1}\right|=\left|\mathbf{v}_{2}\right|=\left|\mathbf{v}_{3}\right| \geqslant\left(D^{\prime}\right)^{1 / 3}
$$

и

$$
\rho(\mathbf{0}, S(F)) \geqslant \frac{\left(D^{\prime}\right)^{1 / 3}}{\sqrt{3}} .
$$

Решетка $\Lambda^{\prime}=\Lambda \cap \operatorname{aff}(S(F))$ двумерна, поэтому $\Lambda$ распадается на двумерные “слои", параллельные $\Lambda^{\prime}$. Поскольку $\operatorname{det} F \leqslant D$, то количество "слоев" между $\operatorname{aff}(F)$ и $\mathbf{0}$ (включая "слой”, содержащий $\mathbf{0}$, и "слой", лежаший в $\operatorname{aff}(F)$ ) не может быть больше чем $D+1$. Стало быть, расстояние $d$ между двумя соседними "слоями” обязано быть не меньше чем $\rho(\mathbf{0}, S(F)) / D$. Учитьвая $(7)$, получаем, что

$$
\operatorname{det} \Lambda^{\prime}=\frac{\operatorname{det} \Lambda}{d}=\frac{1}{d} \leqslant \frac{D \sqrt{3}}{\left(D^{\prime}\right)^{1 / 3}},
$$

откуда легко видеть, что

$$
\left|\mathbf{v}_{\min }\right| \leqslant \sqrt{\frac{2 \operatorname{det} \Lambda^{\prime}}{\sqrt{3}}} \leqslant \frac{\sqrt{2 D}}{\left(D^{\prime}\right)^{1 / 6}},
$$

где $\mathbf{v}_{\min }$ - минимальньй вектор решетки $\Lambda^{\prime}$.

Если в $S(F)$ есть точка решетки $\Lambda^{\prime}$, находящаяся на расстоянии, большем чем $\frac{2}{\sqrt{3}}(D+2)\left|\mathbf{v}_{\min }\right|$ от вершин $S(F)$, то в $S(F)$ найдется $D+1$ точка, лежашие на одной прямой, чего не может быть, так как тогда $\operatorname{det} F \geqslant D+1$. Следовательно, все точки множества $\Lambda^{\prime} \cap S(F)$ сосредоточены в $\varepsilon$-окрестностях вершин $S(F)$, где

$$
\varepsilon=\frac{2}{\sqrt{3}}(D+2)\left|\mathbf{v}_{\min }\right| .
$$

Подставляя (8) в (9) и учитьвая (4) и (6), получаем

$$
\varepsilon \leqslant \frac{\sqrt{2}}{3}\left(D^{\prime}\right)^{1 / 3} \leqslant \frac{\sqrt{2}}{3}\left|\mathbf{v}_{i}\right| .
$$


Если в $\varepsilon$-окрестности каждой вершины $S(F)$ имеются точки множества $\Lambda^{\prime} \cap$ $S(F)$, то легко видеть, что тогда $\operatorname{vol}_{2} F>\frac{1}{9 \sqrt{3}}\left(D^{\prime}\right)^{2 / 3}$. Отсюда получаем следуюшее соотношение: $\operatorname{det} F>\frac{2}{27} D^{\prime}>D$, которое противоречит условию теоремы. Следовательно, можно считать, не ограничивая общности, что множество $\Lambda^{\prime} \cap S(F)$ сосредоточено в $\varepsilon$-окрестностях точек $\mathbf{v}_{1}$ и $\mathbf{v}_{2}$.

Тогда прямая, проходящая через центр симплекса $S(F)$ и точку $\frac{1}{2}\left(\mathbf{v}_{1}+\mathbf{v}_{2}\right)$, пересекает ровно два ребра грани $F$. Возьмем из этих двух ребер ближайшее к центру $S(F)$ и обозначим его $G$. Рассмотрим грань $F^{\prime}$ паруса П, граничашую с $F$ по $G$.

Вспомним, что расстояние от $\Lambda^{\prime}$ до ближайшей точки из $\Lambda \backslash \Lambda^{\prime}$ не меньше чем $\rho(\mathbf{0}, S(F)) / D$. В то же время, грань $F^{\prime}$ обязана содержать треугольник с основанием $G$ и вершиной из множества $\Lambda \backslash \Lambda^{\prime}$. Следовательно, учитывая (6), (7), (10) и то, что длина $G$ больше чем $\sqrt{2}\left|\mathbf{v}_{i}\right|-2 \varepsilon$, получаем, что

$$
\operatorname{vol}_{2} F^{\prime}>\frac{1}{2} \cdot \frac{\sqrt{2}}{3}\left(D^{\prime}\right)^{1 / 3} \frac{\left(D^{\prime}\right)^{1 / 3}}{D \sqrt{3}}=\frac{\left(D^{\prime}\right)^{2 / 3}}{3 \sqrt{6} D} .
$$

Оценим теперь $\rho\left(\mathbf{0}, S\left(F^{\prime}\right)\right)$. Точки $\mathbf{0}, \mathbf{v}_{3}, \frac{1}{3}\left(2 \mathbf{v}_{1}+\mathbf{v}_{3}\right), \frac{1}{3}\left(2 \mathbf{v}_{2}+\mathbf{v}_{3}\right)$ лежат по одну сторону от плоскости aff $\left(F^{\prime}\right)$, в то время как эта плоскость пересекает все ребра конуса $\mathscr{C}_{o}$. Следовательно, расстояние от $\mathbf{0}$ до точек пересечения плоскости aff $\left(F^{\prime}\right)$ с координатньми прямыми $\left\{\lambda \mathbf{v}_{i}: \lambda \in \mathbb{R}\right\}$ строго больше чем $\frac{2}{3}\left|\mathbf{v}_{i}\right|$. Стало быть,

$$
\rho\left(\mathbf{0}, S\left(F^{\prime}\right)\right)>\frac{2}{3} \rho(\mathbf{0}, S(F)) \geqslant \frac{2}{3 \sqrt{3}}\left(D^{\prime}\right)^{1 / 3} .
$$

Из (11) и (12) следует, что

$$
\operatorname{det} F^{\prime}=3 ! \cdot \frac{1}{3} \operatorname{vol}_{2} F^{\prime} \cdot \rho\left(\mathbf{0}, S\left(F^{\prime}\right)\right)>\frac{2 \sqrt{2}}{27} \frac{D^{\prime}}{D}>D,
$$

что противоречит условию теоремы.

Значит, $\operatorname{det} S(F)<D^{\prime}$, что и требовалось доказать.

\section{§7. Доказательство теоремы 3 для произвольного $n$}

В этом параграфе нам понадобятся дополнительные обозначения. Через $B_{n}$ будем обозначать объем $n$-мерного шара, через $S_{o}$ - стандартный $(n-1)$-мерный симплекс в $\mathbb{R}^{n}$, т.е. симплекс с вершинами $(1,0, \ldots, 0),(0,1, \ldots, 0), \ldots,(0,0, \ldots, 1)$. Если $\Delta-k$-мерный симплекс, то через $c(\Delta)$ будем обозначать его центр. Если $F-k$-мерное множество, то через $\operatorname{diam}(F)$ будем обозначать диаметр $F$, через $\partial F$ и int $F$ - соответственно границу и внутренность $F$ относительно $\operatorname{aff}(F)$, а через $R(F)$ - радиус наибольшего $k$-мерного шара, содержашегося в $F$. Через $\rho_{H}(\cdot, \cdot)$ будем обозначать хаусдорфово расстояние между двумя множествами. Напомним, что хаусдорфовым расстоянием между множествами $F, G \subset \mathbb{R}^{n}$ называется inf множества таких $\varepsilon>0$, что $F$ лежит в $\varepsilon$-окрестности $G$ и $G$ лежит в $\varepsilon$-окрестности $F$. Далее, многогранник с вершинами в точках решетки $\Lambda$ будем называть целочисленным относительно $\Lambda$. Целочисленный многогранник будем называть пустьм, если он не содержит точек решетки $\Lambda$, отличных от вершин. Наконец, 
под углом меж ду двумя подпространствами $\mathbb{R}^{n}$, пересекающимися по 0, будем понимать минимальный угол между векторами этих подпространств.

Лемма 6. Пусть $\Lambda \subset \mathbb{R}^{n}-n$-мерная решетка, и пусть плоскость

$$
\pi=\left\{\mathbf{x} \in \mathbb{R}^{n} \mid x_{1}+\cdots+x_{n}=1\right\}
$$

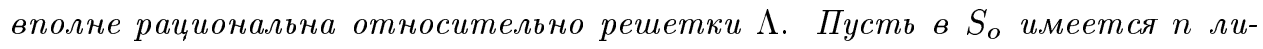
нейно независимых точек из $\Lambda$. Положим $F=\operatorname{conv}\left(S_{o} \cap \Lambda\right)$. Тогда

$$
\operatorname{diam} F>\frac{1}{3 n(n-1)}
$$

ДокАЗАТЕльство. Обозначим $\operatorname{diam} F$ через $\ell$. Многогранник $F$ содержит хотя бы один $(n-1)$-мерный симплекс, целочисленный относительно $\Lambda$. Этот симплекс является базисным для некоторой $(n-1)$-мерной подрешетки $\Lambda^{\prime}$ решетки $\Lambda$. Диаметр этого симплекса не больше чем $\ell$. Следовательно, в шаре радиуса $(n-1) \ell$ с центром в любой точке решетки $\Lambda^{\prime}$ содержится базисный параллелепипед решетки $\Lambda^{\prime}$. Стало быть, в любом шаре радиуса $(n-1) \ell$ с центром, лежащим в плоскости $\pi$, имеется хотя бы одна точка решетки $\Lambda$.

Выделим в $S_{o} n$ "угловых" симплексов:

$$
\Delta_{i}=\operatorname{conv}\left(\frac{2}{3} \mathbf{e}_{i}+\frac{1}{3} \mathbf{e}_{1}, \frac{2}{3} \mathbf{e}_{i}+\frac{1}{3} \mathbf{e}_{2}, \ldots, \frac{2}{3} \mathbf{e}_{i}+\frac{1}{3} \mathbf{e}_{n}\right), \quad i=1,2, \ldots, n
$$

Поскольку $R\left(S_{o}\right)=1 / \sqrt{n(n-1)}>1 / n$, то $R\left(\Delta_{i}\right)>1 /(3 n)$ для каждого $i$.

Если теперь предположить, что $\ell \leqslant 1 /(3 n(n-1))$, то мы получим, что в каждом симплексе $\Delta_{i}$ есть точки решетки $\Lambda$. Но тогда

$$
\ell \geqslant \rho\left(\Delta_{1}, \Delta_{2}\right)=\frac{\sqrt{2}}{3}>\frac{1}{3 n(n-1)}
$$

- противоречие.

ЛЕМма 7. Пусть $F \subset \mathbb{R}^{n-1}-(n-1)$-мерный выпуклый многогранник с вериинами $\mathbf{v}_{1}, \ldots, \mathbf{v}_{m}$. Пусть

$$
\operatorname{vol}_{n-1}(F) \leqslant B_{n-1} \cdot\left(\ell\left(\frac{\lambda}{2}\right)^{2^{n-1}}\right)^{n-1}
$$

где $\ell=\operatorname{diam} F$, а $\lambda-$ произвольное положстельное число, меньиее 1. Тогда можно найти такие точки $\zeta_{1}, \ldots, \zeta_{m} \in \mathbb{R}^{n-1}$, что

$$
\max _{1 \leqslant i \leqslant m}\left|\mathbf{v}_{i}-\boldsymbol{\zeta}_{i}\right| \leqslant \lambda R(\Gamma)
$$

әде $\Gamma=\operatorname{conv}\left(\boldsymbol{\zeta}_{1}, \ldots, \boldsymbol{\zeta}_{m}\right)$, причем $\operatorname{dim} \Gamma=k \leqslant n-2$. 
ДокАЗАТЕЛЬСтво. Допустим, что таких точек не существует. Не ограничивая общности, будем считать, что $\left|\mathbf{v}_{1}-\mathbf{v}_{2}\right|=\ell$.

Положим $I_{1}=\operatorname{conv}\left(\mathbf{v}_{1}, \mathbf{v}_{2}\right)$. Если $F$ находится в окрестности радиуса $\lambda R\left(I_{1}\right)=$ $\lambda \ell / 2$ прямой aff $\left(I_{1}\right)$, то на этой прямой мы можем выбрать точки $\zeta_{1}=\mathbf{v}_{1}, \zeta_{2}=\mathbf{v}_{2}$, $\zeta_{3}, \ldots, \zeta_{m}$ так, чтобы для всех $i$ выполнялось

$$
\left|\mathbf{v}_{i}-\zeta_{i}\right| \leqslant \lambda R\left(I_{1}\right) \leqslant \lambda R\left(\operatorname{conv}\left(\zeta_{1}, \ldots, \zeta_{m}\right)\right)
$$

Но мы предположили противное. Стало быть, в $F$ найдется точка, лежашая на расстоянии, большем чем $\lambda R\left(I_{1}\right)$, от aff $\left(I_{1}\right)$. Не ограничивая обшности, будем считать, что это точка $\mathbf{v}_{3}$.

Положим $I_{2}=\operatorname{conv}\left(\mathbf{v}_{1}, \mathbf{v}_{2}, \mathbf{v}_{3}\right)$. По аналогичньм соображениям в $F$ найдется точка, лежашая на расстоянии, большем чем $\lambda R\left(I_{2}\right)$, от плоскости $\operatorname{aff}\left(I_{2}\right)$. Опять же, не ограничивая обшности, будем считать, что это точка $\mathbf{v}_{4}$.

Описанное построение мы можем продолжить, пока не получим симплекс

$$
I_{n-1}=\operatorname{conv}\left(\mathbf{v}_{1}, \ldots, \mathbf{v}_{n}\right)
$$

очевидно, содержашийся в $F$. Однако из построения $I_{n-1}$ следует, что

$$
R\left(I_{n-1}\right)>\ell\left(\frac{\lambda}{2}\right)^{2^{n-1}}
$$

Докажем это. Точнее, докажем по индукции, что $R\left(I_{k}\right) \geqslant \ell(\lambda / 2)^{2^{k}-1}$ для всех $k=1,2, \ldots, n-1$. Основание индукции при $k=1$ очевидно. Пусть $B-k$-мерный шар радиуса $\ell(\lambda / 2)^{2^{k}-1}$, содержащийся в $I_{k}$. Тогда $I_{k+1}$ содержит $(k+1)$-мерньй конус с основанием $B$ и вершиной $\mathbf{v}_{k+2}$. Причем, во-первых,

$$
\rho\left(\mathbf{v}_{k+2}, \operatorname{aff}\left(I_{k}\right)\right) \geqslant \lambda R\left(I_{k}\right) \geqslant \lambda \ell\left(\frac{\lambda}{2}\right)^{2^{k}-1}
$$

а во-вторых, расстояние от $\mathbf{v}_{k+2}$ до любой точки шара $B$ не превышает $\ell$. Из элементарных геометрических соображений заключаем, что конус содержит шар радиуса $\ell(\lambda / 2)^{2^{k+1}-1}$. То есть $R\left(I_{k+1}\right) \geqslant \ell(\lambda / 2)^{2^{k+1}-1}$, а поскольку $\lambda<1$, то $R\left(I_{n-1}\right)>\ell(\lambda / 2)^{2^{n-1}}$.

Следовательно,

$$
\operatorname{vol}_{n-1}(F)>B_{n-1}\left(\ell\left(\frac{\lambda}{2}\right)^{2^{n-1}}\right)^{n-1},
$$

что противоречит условию леммы.

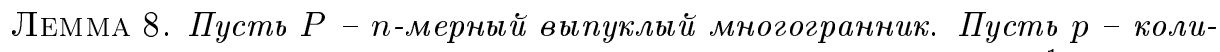
чество его $(n-1)$-мерных граней. Тогда $P$ можно разбить на $p^{n-1}$ симплексов. 
ДокАЗАТЕЛЬСТво. Будем доказывать по индукции. Для $n=1$ утверждение очевидно. Пусть теперь $n \geqslant 2$. Возьмем произвольную точку $a \in \operatorname{int} P$ и разобьем $P$ следующим образом:

$$
P=\bigcup_{F} \operatorname{conv}(F \cup\{a\}),
$$

где $F$ пробегает множество всех $(n-1)$-мерных граней $P$. К каждой такой грани $F$ применимо предположение индукции, поскольку количество $(n-2)$-мерных граней многогранника $F$ не превосходит $p$. То есть каждую $(n-1)$-мерную грань $F$ многогранника $P$ можно разбить на $p^{n-2}(n-1)$-мерных симплексов. Но тогда весь многогранник $P$ разбивается на $p^{n-1} n$-мерных симплексов.

Лемма 9. Пусть $F=\operatorname{conv}\left(\mathbf{v}_{1}, \ldots, \mathbf{v}_{m}\right) \subset \mathbb{R}^{n}, \operatorname{dim} F=k \geqslant 1$. Тогда в $F$ можно найти $k$-мерный симплекс $\Delta$ объема не менее

$$
8^{-m^{n}} \operatorname{vol}_{k} F
$$

и такой, что любой симплекс с вершинами из множества $V=\left\{\mathbf{v}_{1}, \ldots, \mathbf{v}_{m}\right\}$ либо не пересекается $c$ int $\Delta$, либо содержит $\Delta$.

ДоказАтельство. Очевидно, $F$ содержит не более $m^{k}$ различных $k$-мерных симплексов с вершинами из множества $V$. Следовательно, разбиение $F$, получающееся путем пересечения всех таких симплексов, содержит не более $2^{m^{k}}$ ячеек. Причем каждая такая ячейка представляет собой выпуклый $k$-мерный многогранник, у которого количество $(k-1)$-мерных граней не превосходит $(k+1) m^{k}$. По лемме 8 каждую ячейку можно разбить на

$$
\left((k+1) m^{k}\right)^{k-1}
$$

симплексов. В итоговом разбиении $F$ будет не более чем

$$
2^{m^{k}}\left((k+1) m^{k}\right)^{k-1} \leqslant 2^{m^{n}}(n+1)^{n-1} m^{n(n-1)} \leqslant 8^{m^{n}}
$$

симплексов. Выбрав из этого разбиения симплекс с наибольшим объемом, получим искомый симплекс.

Лемма 10. Пусть $P-k$-мерный выпуклыцй многогранник, $\Delta \subset P-k$-мерньий симплекс. Тогда

$$
\frac{R(\Delta)}{R(P)} \geqslant \frac{\operatorname{vol}_{k}(\Delta)}{\operatorname{vol}_{k}(P)}
$$

ДокАЗАТЕЛЬСТво. Поскольку $\Delta$ - симплекс, а $P$ - выпуклый многогранник, то

$$
\begin{aligned}
\operatorname{vol}_{k} \Delta & =\frac{1}{k} R(\Delta) \operatorname{vol}_{k-1} \partial \Delta, \\
\operatorname{vol}_{k} P & \geqslant \frac{1}{k} R(P) \operatorname{vol}_{k-1} \partial P .
\end{aligned}
$$

Следовательно,

$$
\frac{R(\Delta)}{R(P)} \geqslant \frac{\operatorname{vol}_{k}(\Delta)}{\operatorname{vol}_{k}(P)} \cdot \frac{\operatorname{vol}_{k-1}(\partial P)}{\operatorname{vol}_{k-1}(\partial \Delta)} \geqslant \frac{\operatorname{vol}_{k}(\Delta)}{\operatorname{vol}_{k}(P)}
$$


Лемма 11. Пусть $\Delta-k$-мерный симплекс. Тогда

$$
\rho(c(\Delta), \partial \Delta)>\frac{2}{k+1} R(\Delta)
$$

ДокАЗАТЕЛЬство. Если $F$ - произвольная $(k-1)$-мерная грань $\Delta$, то

$$
\operatorname{vol}_{k}(\operatorname{conv}(F \cup\{c(\Delta)\}))=\frac{1}{k+1} \operatorname{vol}_{k}(\Delta)=\frac{1}{k+1} \cdot \frac{1}{k} \operatorname{vol}_{k-1}(\partial \Delta) \cdot R(\Delta) .
$$

С другой стороны, если в качестве $F$ взять грань $\Delta$, ближайшую к $c(\Delta)$, то

$\operatorname{vol}_{k}(\operatorname{conv}(F \cup\{c(\Delta)\}))=\frac{1}{k} \operatorname{vol}_{k-1}(F) \cdot \rho(c(\Delta), \partial \Delta)<\frac{1}{2 k} \operatorname{vol}_{k-1}(\partial \Delta) \cdot \rho(c(\Delta), \partial \Delta)$.

Следовательно,

$$
\frac{1}{2 k} \operatorname{vol}_{k-1}(\partial \Delta) \cdot \rho(c(\Delta), \partial \Delta)>\frac{1}{k+1} \cdot \frac{1}{k} \operatorname{vol}_{k-1}(\partial \Delta) \cdot R(\Delta),
$$

откуда видим, что, действительно, $\rho(c(\Delta), \partial \Delta)>\frac{2}{k+1} R(\Delta)$.

Лемма 12. Пусть $F$ - k-мерное выпуклое множество, содержащее $k$-мерный симплекс $\Delta$ такой, что $\operatorname{vol}_{k} \Delta \geqslant \varkappa \operatorname{vol}_{k} F$. Пусть точки $\mathbf{a}_{1}, \mathbf{a}_{2} \in \partial F$ лежат на одной прямой с $c(\Delta)$. Тогда

$$
\frac{\left|\mathbf{a}_{1}-c(\Delta)\right|}{\left|\mathbf{a}_{2}-c(\Delta)\right|} \geqslant \frac{\varkappa}{k(k+1)} \text {. }
$$

ДОКАЗАТЕЛЬСТво. Для $i=1,2$ положим $\mathbf{b}_{i}=\partial \Delta \cap\left[\mathbf{a}_{i}, c(\Delta)\right]$ и обозначим через $G$ произвольную $(k-1)$-мерную грань симплекса $\Delta$, содержашую точку $\mathbf{b}_{2}$. Тогда

$$
\begin{aligned}
\frac{\left|\mathbf{b}_{2}-c(\Delta)\right|}{\left|\mathbf{a}_{2}-c(\Delta)\right|} & =\frac{\left|\mathbf{b}_{2}-c(\Delta)\right| \cdot \operatorname{vol}_{k-1}(G)}{\left|\mathbf{a}_{2}-c(\Delta)\right| \cdot \operatorname{vol}_{k-1}(G)}=\frac{\operatorname{vol}_{k}(\operatorname{conv}(G \cup\{c(\Delta)\}))}{\operatorname{vol}_{k}\left(\operatorname{conv}\left(G \cup\left\{\mathbf{a}_{2}, c(\Delta)\right\}\right)\right)} \\
& \geqslant \frac{1}{k+1} \frac{\operatorname{vol}_{k} \Delta}{\operatorname{vol}_{k} F} \geqslant \frac{\varkappa}{k+1} .
\end{aligned}
$$

С другой стороны,

$$
\frac{\left|\mathbf{b}_{1}-c(\Delta)\right|}{\left|\mathbf{b}_{2}-c(\Delta)\right|} \geqslant \frac{1}{k}
$$

поскольку $c(\Delta)$ - центр масс симплекса $\Delta$.

Следовательно,

$$
\frac{\left|\mathbf{a}_{1}-c(\Delta)\right|}{\left|\mathbf{a}_{2}-c(\Delta)\right|} \geqslant \frac{\left|\mathbf{b}_{1}-c(\Delta)\right|}{\left|\mathbf{b}_{2}-c(\Delta)\right|} \cdot \frac{\left|\mathbf{b}_{2}-c(\Delta)\right|}{\left|\mathbf{a}_{2}-c(\Delta)\right|} \geqslant \frac{\varkappa}{k(k+1)} .
$$

Напомним, что через $\rho_{H}(\cdot, \cdot)$ мы обозначаем хаусдорфово расстояние между двумя множествами. 
Лемма 13. Пусть $F, F^{\prime} \subset \mathbb{R}^{k}-k$-мерные выпуклье компактные множества. Пусть $\mathbf{0} \in F$ и $\rho(\mathbf{0}, \partial F) \geqslant 2 \rho_{H}\left(F, F^{\prime}\right)$. Тогда

$$
\frac{1}{2} F \subseteq F^{\prime} \subseteq \frac{3}{2} F
$$

ДокАЗАтЕЛЬСтво. Рассмотрим произвольную точку $\mathbf{a} \in \partial F$. Шар радиуса $\rho(\mathbf{0}, \partial F)$ с центром в $\mathbf{0}$ обозначим $B$. Очевидно, $\operatorname{conv}(B \cup\{\mathbf{a}\}) \subseteq F$ и $\operatorname{conv}\left(\frac{3}{2} B \cup\right.$ $\left.\left\{\frac{3}{2} \mathbf{a}\right\}\right) \subseteq \frac{3}{2} F$, откуда легко видеть, что $F$ содержит шар радиуса $\frac{1}{2} \rho(\mathbf{0}, \partial F)$ с центром в $\mathbf{a} / 2$, а $\frac{3}{2} F$ содержит шар радиуса $\frac{1}{2} \rho(\mathbf{0}, \partial F)$ с центром в $\mathbf{a}$. Следовательно,

$$
\begin{aligned}
\rho\left(\frac{1}{2} F, \partial F\right) & =\frac{1}{2} \rho(\mathbf{0}, \partial F), \\
\rho\left(F, \partial\left(\frac{3}{2} F\right)\right) & =\frac{1}{2} \rho(\mathbf{0}, \partial F) .
\end{aligned}
$$

Допустим, что $\frac{1}{2} F \nsubseteq F^{\prime}$, и рассмотрим $\mathbf{x} \in\left(\frac{1}{2} F\right) \backslash F^{\prime}$. Поскольку $F^{\prime}$ выпукло и замкнуто, то найдется $(k-1)$-мерная плоскость $\pi$, строго отделяющая $\mathbf{x}$ от $F^{\prime}$. Рассмотрим прямую $\pi^{\perp}$, ортогональную $\pi$ и проходящую через $\mathbf{x}$. Точку из множества $\pi^{\perp} \cap \partial F$, находящуюся относительно плоскости $\pi$ в одном полупространстве с точкой $\mathbf{x}$, обозначим $\mathbf{y}$. Учитьвая (13), получаем

$$
\rho\left(\mathbf{y}, F^{\prime}\right)>\rho(\mathbf{y}, \pi)>\rho(\mathbf{y}, \mathbf{x}) \geqslant \rho\left(\frac{1}{2} F, \partial F\right)=\frac{1}{2} \rho(\mathbf{0}, \partial F) \geqslant \rho_{H}\left(F, F^{\prime}\right),
$$

что противоречит определению хаусдорфова расстояния. Следовательно,

$$
\frac{1}{2} F \subseteq F^{\prime}
$$

Допустим теперь, что $F^{\prime} \nsubseteq \frac{3}{2} F$, и рассмотрим $\mathbf{x} \in F^{\prime} \backslash\left(\frac{3}{2} F\right)$. По определению хаусдорфова расстояния в $F$ найдется такая точка $\mathbf{y}$, что $\rho(\mathbf{x}, \mathbf{y}) \leqslant \rho_{H}\left(F, F^{\prime}\right) \leqslant$ $\frac{1}{2} \rho(\mathbf{0}, \partial F)$. Поскольку $\mathbf{x} \notin \frac{3}{2} F$, а $\mathbf{y} \in F \subset \frac{3}{2} F$, то на отрезке $[\mathbf{x}, \mathbf{y}]$ найдется точка $\mathbf{z} \in \partial\left(\frac{3}{2} F\right)$, причем из замкнутости $F$ следует, что $\mathbf{z} \neq \mathbf{x}$. Следовательно,

$$
\rho\left(F, \partial\left(\frac{3}{2} F\right)\right) \leqslant \rho(\mathbf{y}, \mathbf{z})<\frac{1}{2} \rho(\mathbf{0}, \partial F)
$$

что противоречит формуле (14). Стало быть,

$$
F^{\prime} \subseteq \frac{3}{2} F
$$


Лемма 14. Пусть $\mathbb{R}^{n}=L_{1} \oplus L_{2}, L_{1} \perp L_{2}, \operatorname{dim} L_{1}=k \leqslant n-1, \operatorname{dim} L_{2}=$ $n-k$. Пусть $F \subset \mathbb{R}^{n}$ - n-мерное выпуклое компактное множество. Пусть $\Gamma \subset L_{1}-k$-мерное выпуклое компактное множество, содержащее $\mathbf{0}$. Пусть $\rho(\mathbf{0}, \partial \Gamma) \geqslant 2 \rho_{H}(F, \Gamma)$, и пусть для любъх двух точек $\mathbf{a}_{1}, \mathbf{a}_{2} \in \partial \Gamma$, лежащих на одной прямой с точкой $\mathbf{0}$, выполняется $\left|\mathbf{a}_{1}\right| \geqslant \gamma\left|\mathbf{a}_{2}\right|$, где $\gamma>0$. (Очевидно, $\gamma$ не моэсет бьть больше 1.) Тогда

$$
\operatorname{vol}_{k} \Gamma \cdot \operatorname{vol}_{n-k}\left(F \cap L_{2}\right)>\left(\frac{\gamma}{8}\right)^{n} \operatorname{vol}_{n} F
$$

ДокАЗАТЕльство. Обозначим через $\mathscr{P}_{F}$ ортогональную проекцию $F$ на $L_{1}$ и положим $f(\mathbf{x})=\operatorname{vol}_{n-k}\left(F \cap\left\{\mathbf{x}+L_{2}\right\}\right)$ для всех $\mathbf{x} \in L_{1}$. Точку, на которой достигается максимум функции $f$, обозначим $\mathbf{x}_{\max }$.

Очевидно, $f\left(\mathbf{x}_{\max }\right) \operatorname{vol}_{k}\left(\mathscr{P}_{F}\right) \geqslant \operatorname{vol}_{n} F$. С другой стороны, имеет место неравенство $\rho(\mathbf{0}, \partial \Gamma) \geqslant 2 \rho_{H}\left(\mathscr{P}_{F}, \Gamma\right)$, поскольку $\mathscr{P}_{F}$ - ортогональная проекция $F$ на $L_{1}$. Следовательно, применив лемму 13, получим, что $\mathscr{P}_{F} \subseteq \frac{3}{2} \Gamma$ и $\operatorname{vol}_{k}\left(\mathscr{P}_{F}\right) \leqslant$ $\left(\frac{3}{2}\right)^{k} \operatorname{vol}_{k} \Gamma<2^{k} \operatorname{vol}_{k} \Gamma$. Стало быть,

$$
\operatorname{vol}_{k} \Gamma \cdot f\left(\mathbf{x}_{\max }\right)>2^{-k} \operatorname{vol}_{n} F
$$

Если $\mathbf{x}_{\max }=\mathbf{0}$, то $f\left(\mathbf{x}_{\max }\right)=\operatorname{vol}_{n-k}\left(F \cap L_{2}\right)$, т.е. в этом случае лемма доказана. Если же $\mathbf{x}_{\max } \neq \mathbf{0}$, то возьмем такие $\mu_{1}, \mu_{2}, \mu_{3}>0$, что $\mathbf{y}=-\mu_{1} \mathbf{x}_{\max } \in \partial \mathscr{P}_{F}$, $\mathbf{z}=-\mu_{2} \mathbf{x}_{\max } \in \partial \Gamma, \mathbf{w}=\mu_{3} \mathbf{x}_{\max } \in \partial \Gamma$. Из леммы 13 следует, что $\mu_{1} \geqslant \frac{1}{2} \mu_{2}$ и $\mu_{3} \geqslant \frac{2}{3}$. Следовательно,

$$
\frac{\left|\mathbf{x}_{\max }\right|}{|\mathbf{y}|} \leqslant \frac{3|\mathbf{w}|}{|\mathbf{z}|} \leqslant \frac{3}{\gamma}
$$

В силу замкнутости $F$ множество $F \cap\left\{\mathbf{y}+L_{2}\right\}$ непусто. Пусть $\mathbf{y}^{\prime}$ - произвольная точка из $F \cap\left\{\mathbf{y}+L_{2}\right\}$. Поскольку $F$ вьпукло, $\operatorname{conv}\left(\{\mathbf{y}\} \cup\left(F \cap\left\{\mathbf{y}+L_{2}\right\}\right)\right) \subseteq F$. Следовательно,

$$
\operatorname{vol}_{n-k}\left(F \cap L_{2}\right) \geqslant \operatorname{vol}_{n-k}\left(F \cap\left(\mathbf{x}_{\text {max }}+L_{2}\right)\right) \cdot\left(\frac{|\mathbf{y}|}{|\mathbf{y}|+\left|\mathbf{x}_{\max }\right|}\right)^{n-k}
$$

Из неравенств (16) и (17) получаем, что

$f(\mathbf{0}) \geqslant f\left(\mathbf{x}_{\max }\right)\left(\frac{|\mathbf{y}|}{|\mathbf{y}|+\left|\mathbf{x}_{\max }\right|}\right)^{n-k}=\left(\frac{\gamma}{3+\gamma}\right)^{n-k} f\left(\mathbf{x}_{\max }\right)>\left(\frac{\gamma}{8}\right)^{n-k} f\left(\mathbf{x}_{\max }\right)$.

Из неравенств (15) и (18) следует, что

$\operatorname{vol}_{k} \Gamma \cdot \operatorname{vol}_{n-k}\left(F \cap L_{2}\right)=\operatorname{vol}_{k} \Gamma \cdot f(\mathbf{0})>\gamma^{n-k} 2^{2 k-3 n} \operatorname{vol}_{n} F \geqslant \gamma^{n} 2^{-3 n} \operatorname{vol}_{n} F$. 
Лемма 15. Пусть $\mathbb{R}^{n}=L_{1} \oplus L_{2}, \operatorname{dim} L_{1}=k \leqslant n-1, \operatorname{dim} L_{2}=n-k$.

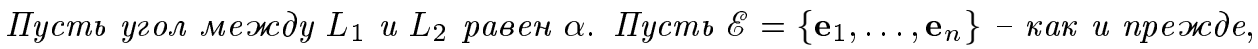
стандартный ортонормированный базис в $\mathbb{R}^{n}$, причем $\left\{\mathbf{e}_{1}, \ldots, \mathbf{e}_{k}\right\}-$ базис в $L_{1}$. Рассмотрим такие $\mathbf{f}_{i} \in L_{2}, i=1, \ldots, n-k$, ито $\mathbf{f}_{i}-\mathbf{e}_{k+i} \in L_{1}$. Рассмотрим оператор $A: \mathbb{R}^{n} \rightarrow \mathbb{R}^{n}$ такой, что $A\left(\mathbf{e}_{i}\right)=\mathbf{e}_{i} \partial л я i=1, \ldots, k$ u $A\left(\mathbf{e}_{k+i}\right)=\mathbf{f}_{i}$ для $i=1, \ldots, n-k$. Тогда для всех $\mathbf{x} \in \mathbb{R}^{n}$ имеют место неравенства

$$
\frac{\sin \alpha}{n}|\mathbf{x}|<|A(\mathbf{x})|<\frac{n}{\sin \alpha}|\mathbf{x}|
$$

и для всех $\mathbf{x} \in L_{2}$ выполняется

$$
|A(\mathbf{x})| \geqslant \sin \alpha|\mathbf{x}| .
$$

ДокаЗательство. Рассмотрим куб $\left\{\mathbf{x} \in \mathbb{R}^{n}|| x_{i} \mid \leqslant 1, i=1, \ldots, n\right\}$. Оператор $A$ переводит этот куб в некоторый параллелепипед, центрами $(n-1)$-мерных граней которого являются точки $\mathbf{e}_{1}, \ldots, \mathbf{e}_{k}, \mathbf{f}_{1}, \ldots, \mathbf{f}_{n-k}$. Угол между $\mathbf{e}_{k+i}$ и $\mathbf{f}_{i}$ больше, либо равен $\alpha$. Следовательно, расстояние от любой точки этого параллелепипеда до $\mathbf{0}$ меньше чем $k+(n-k) / \sin \alpha$. Отсюда заключаем, что если $|\mathbf{x}|=1$, то $|A(\mathbf{x})|<k+(n-k) / \sin \alpha \leqslant n / \sin \alpha$ или, иначе говоря,

$$
|A(\mathbf{x})|<\frac{n}{\sin \alpha}|\mathbf{x}|
$$

для всех $\mathbf{x} \in \mathbb{R}^{n}$.

Положим теперь $\mathbf{f}_{i}^{\prime}=2 \mathbf{e}_{k+i}-\mathbf{f}_{i}, i=1, \ldots, n-k$. Тогда $A\left(\mathbf{f}_{i}^{\prime}\right)=\mathbf{e}_{k+i}$, поскольку $\mathbf{e}_{k+i}-\mathbf{f}_{i} \in L_{1}$, и угол между $\mathbf{e}_{k+i}$ и $\mathbf{f}_{i}^{\prime}$ равен углу между $\mathbf{e}_{k+i}$ и $\mathbf{f}_{i}$, поскольку $\mathbf{e}_{k+i} \perp L_{1}$ для всех $i=1, \ldots, n-k$. Следовательно, мы можем применить для оператора $A^{-1}$ те же рассуждения, что и для $A$, и получить, что

$$
\left|A^{-1}(\mathbf{x})\right|<\frac{n}{\sin \alpha}|\mathbf{x}|
$$

для всех $\mathbf{x} \in \mathbb{R}^{n}$.

Неравенства (21) и (22) дают неравенство (19).

Далее, угол между любыми двумя векторами из $L_{1}$ и $L_{2}$ больше, либо равен $\alpha$, a оператор $A$ проектирует $L_{2}$ ортогонально на $L_{2}^{\prime}$ вдоль $L_{1}$. Следовательно, $|A(\mathbf{x})| \geqslant$ $\sin \alpha|\mathbf{x}|$ для всех $\mathbf{x} \in L_{2}$.

Лемма 16. Пусть $\mathbb{R}^{n}=L_{1} \oplus L_{2}, \operatorname{dim} L_{1}=k \leqslant n-1, \operatorname{dim} L_{2}=n-k$. Пусть угол межсуу $L_{1}$ и $L_{2}$ равен $\alpha$. Пусть $F \subset \mathbb{R}^{n}-n$-мерное выпуклое компактное множество. Пусть $\Gamma \subset L_{1}-k$-мерное выпуклое компактное множество, содержащее $\mathbf{0}$. Пусть

$$
\rho(\mathbf{0}, \partial \Gamma) \geqslant \frac{2 n}{\sin \alpha} \rho_{H}(F, \Gamma),
$$

и пусть для любъх двух точек $\mathbf{a}_{1}, \mathbf{a}_{2} \in \partial \Gamma$, лежащих на одной прямой с точкой $\mathbf{0}$, выполняется $\left|\mathbf{a}_{1}\right| \geqslant \gamma\left|\mathbf{a}_{2}\right|$, где $\gamma>0$. Тогда

$$
\operatorname{vol}_{k} \Gamma \cdot \operatorname{vol}_{n-k}\left(F \cap L_{2}\right)>\left(\frac{\gamma}{8}\right)^{n} \operatorname{vol}_{n} F .
$$


ДоказАТЕЛЬСтво. Обозначим через $L_{2}^{\prime}$ ортогональное дополнение к $L_{1}$ и рассмотрим оператор $A$ из леммы 15 . По этой лемме

$$
|A(\mathbf{x})-A(\mathbf{y})|<\frac{n}{\sin \alpha}|\mathbf{x}-\mathbf{y}|
$$

для всех $\mathbf{x}, \mathbf{y} \in \mathbb{R}^{n}$.

Следовательно, $\rho(\mathbf{0}, \partial \Gamma)>2 \rho_{H}(A(F), \Gamma)$, т.е. мы можем применить лемму 14 и получить, что

$$
\operatorname{vol}_{k} \Gamma \cdot \operatorname{vol}_{n-k}\left(A(F) \cap L_{2}^{\prime}\right)>\left(\frac{\gamma}{8}\right)^{n} \operatorname{vol}_{n} F
$$

Ho $\operatorname{vol}_{n-k}\left(A(F) \cap L_{2}^{\prime}\right) \leqslant \operatorname{vol}_{n-k}\left(F \cap L_{2}\right)$, так как $A$ проектирует $L_{2}$ ортогонально на $L_{2}^{\prime}$. Стало быть,

$$
\operatorname{vol}_{k} \Gamma \cdot \operatorname{vol}_{n-k}\left(F \cap L_{2}\right)>\left(\frac{\gamma}{8}\right)^{n} \operatorname{vol}_{n} F .
$$

Лемма 17. Пусть $G, \Delta \subseteq \mathbb{R}^{n}-k$-мерные выпуклье компактные множества. Пусть $\Delta$ содержится в в-окрестности $G$. Пусть $\mathbf{0} \in \Delta u \rho(\mathbf{0}, \partial \Delta) \geqslant 2 \varepsilon$. Тогда

$$
\operatorname{vol}_{k} G \geqslant 2^{-k} \operatorname{vol}_{k} \Delta
$$

Доказательство. Обозначим через $\mathscr{P}_{G}$ ортогональную проекцию $G$ на $\operatorname{aff}(\Delta)$. Тогда $\Delta$ содержится и в $\varepsilon$-окрестности $\mathscr{P}_{G}$. Рассмотрим произвольную точку $\mathbf{x} \in \Delta$ такую, что $\rho(\mathbf{x}, \partial \Delta) \geqslant \frac{1}{2} \rho(\mathbf{0}, \partial \Delta)$. Если $\mathbf{x} \notin \mathscr{P}_{G}$, то в $\operatorname{aff}(\Delta)$ найдется $(k-1)$-мерная плоскость, строго отделяющая $\mathbf{x}$ от $\mathscr{P}_{G}$. Но $\rho(\mathbf{x}, \partial \Delta) \geqslant$ $\frac{1}{2} \rho(\mathbf{0}, \partial \Delta) \geqslant \varepsilon$, значит, в $\partial \Delta$ можно найти такую точку $\mathbf{y}$, что $\rho\left(\mathbf{y}, \mathscr{P}_{G}\right)>\varepsilon$, чего не может быть. Стало быть,

$$
\mathscr{P}_{G} \supseteq \Delta^{\prime}=\left\{\mathbf{x} \in \Delta \mid \rho(\mathbf{x}, \partial \Delta) \geqslant \frac{1}{2} \rho(\mathbf{0}, \partial \Delta)\right\} .
$$

Докажем, что $\Delta^{\prime}$ содержит $\frac{1}{2} \Delta$. Для этого рассмотрим произвольную точку $\mathbf{a} \in \partial \Delta$ и обозначим через $B$ шар радиуса $\rho(\mathbf{0}, \partial \Delta)$ с центром в $\mathbf{0}$. Очевидно, $((1-\mu) B+\mu \mathbf{a}) \subset \operatorname{conv}(B \cup\{\mathbf{a}\}) \subseteq \Delta$ для всех $\mu \in[0,1]$. Отсюда легко видеть, что $\frac{1}{2} \Delta \subset \Delta^{\prime}$.

Следовательно,

$$
\operatorname{vol}_{k} G \geqslant \operatorname{vol}_{k} \mathscr{P}_{G} \geqslant 2^{-k} \operatorname{vol}_{k} \Delta
$$

Лемма 18. Пусть $\mathbb{R}^{n}=L_{1} \oplus L_{2}, L_{1} \perp L_{2}, \operatorname{dim} L_{1}=k \leqslant n-1, \operatorname{dim} L_{2}=n-k$. Пусть $F \subset L_{1}-k$-мерное выпуклое компактное множество. Пусть $G \subset L_{2}$ - $(n-k)$-мерное выпуклое компактное множество. Пусть к тому же $F$ u $G$ содержат $\mathbf{0}$. Тогда

$$
\operatorname{vol}_{n}(\operatorname{conv}(F \cup G))=\frac{1}{\left(\begin{array}{c}
n-1 \\
k
\end{array}\right)} \operatorname{vol}_{k} F \cdot \operatorname{vol}_{n-k} G .
$$


ДоКАЗАТЕЛЬСТво. Поскольку любое выпуклое множество можно сколь угодно хорошо приблизить выпуклым многогранником соответствуюшей размерности, достаточно доказать лемму для выпуклых многогранников. Далее, в силу аддитивности объема достаточно доказать лемму для случая, когда $F$ и $G$ являются соответственно $k$-мерным и $(n-k)$-мерным симплексами, у каждого из которых одна из вершин совпадает с 0. А эта задача легко сводится к случаю, когда $F=\operatorname{conv}\left(\mathbf{0}, \mathbf{e}_{1}, \ldots, \mathbf{e}_{k}\right)$, a $G=\operatorname{conv}\left(\mathbf{0}, \mathbf{e}_{k+1}, \ldots, \mathbf{e}_{n}\right)$, где $\left\{\mathbf{e}_{1}, \ldots, \mathbf{e}_{n}\right\}$-как и прежде, стандартный ортонормированный базис в $\mathbb{R}^{n}$. Для таких $F$ и $G$ имеем

$$
\frac{\operatorname{vol}_{n}(\operatorname{conv}(F \cup G))}{\operatorname{vol}_{k} F \cdot \operatorname{vol}_{n-k} G}=\frac{\frac{1}{n !}}{\frac{1}{k !} \cdot \frac{1}{(n-k) !}}=\frac{1}{\left(\begin{array}{c}
n-1 \\
k
\end{array}\right)} \text {. }
$$

Лемма 19. Пусть $\mathbb{R}^{n}=L_{1} \oplus L_{2}, L_{1} \perp L_{2}, \operatorname{dim} L_{1}=k \leqslant n-2, \operatorname{dim} L_{2}=$ $n-k$. Пусть $G \subset \mathbb{R}^{n}-(n-1)$-мерное выпуклое компактное мнохество. Пусть $\Delta \subset L_{1}-k$-мерное выпуклое компактное множсество, содержащее $\mathbf{0}$ и лежсащее в в-окрестности множества $G$. Пусть $\rho(\mathbf{0}, \partial \Delta) \geqslant 2 \varepsilon$. Тогда

$$
\operatorname{vol}_{n-1} G>(2 n)^{-k} \operatorname{vol}_{k} \Delta \cdot \operatorname{vol}_{n-k-1}\left(G \cap L_{2}\right)
$$

ДоказАтельство. Обозначим через $\mathscr{P}_{G}$ ортогональную проекцию $G$ на $L_{1}$, возьмем произвольную точку $\mathbf{x}_{o} \in G \cap L_{2}$ и рассмотрим множество

$$
G^{\prime}=\operatorname{conv}\left(\left\{G-\mathbf{x}_{o}\right\} \cap L_{2}, \mathscr{P}_{G}\right)
$$

Пользуясь леммой 18, получаем

$$
\begin{aligned}
\operatorname{vol}_{n-1} G^{\prime} & =\frac{1}{\left(\begin{array}{c}
n-1 \\
k
\end{array}\right)} \operatorname{vol}_{k} \mathscr{P}_{G} \cdot \operatorname{vol}_{n-k-1}\left(G \cap L_{2}\right) \\
& >n^{-k} \operatorname{vol}_{k} \mathscr{P}_{G} \cdot \operatorname{vol}_{n-k-1}\left(G \cap L_{2}\right) .
\end{aligned}
$$

Из выпуклости $G$ и ортогональности $L_{1}$ и $L_{2}$ следует, что $\operatorname{vol}_{n-1} G \geqslant \operatorname{vol}_{n-1} G^{\prime}$. Покажем, что $\mathscr{P}_{G} \supseteq \frac{1}{2} \Delta$. Как и в лемме 13 , рассмотрим произвольную точку $\mathbf{a} \in \partial \Delta$. Шар радиуса $\rho(\mathbf{0}, \partial \Delta)$ с центром в $\mathbf{0}$ обозначим $B$. Тогда $\left(\frac{1}{2} B+\frac{1}{2} \mathbf{a}\right) \subset$ $\operatorname{conv}(B \cup\{\mathbf{a}\}) \subseteq \Delta$. Следовательно,

$$
\rho\left(\frac{1}{2} \Delta, \partial \Delta\right)=\frac{1}{2} \rho(\mathbf{0}, \partial \Delta) .
$$

Допустим, что $\mathscr{P}_{G} \nsupseteq \frac{1}{2} \Delta$, и рассмотрим $\mathbf{x} \in\left(\frac{1}{2} \Delta\right) \backslash \mathscr{P}_{G}$. Поскольку $\mathscr{P}_{G}$ выпукло и замкнуто, то найдется $(k-1)$-мерная плоскость $\pi$, строго отделяющая $\mathbf{x}$ от $\mathscr{P}_{G}$. Рассмотрим прямую $\pi^{\perp}$, ортогональную $\pi$ и проходящую через $\mathbf{x}$. Точку из множества $\pi^{\perp} \cap \partial \Delta$, находящуюся относительно плоскости $\pi$ в одном полупространстве с точкой х, обозначим у. Учитывая (24), получаем

$$
\rho\left(\mathbf{y}, \mathscr{P}_{G}\right)>\rho(\mathbf{y}, \pi)>\rho(\mathbf{y}, \mathbf{x}) \geqslant \rho\left(\frac{1}{2} \Delta, \partial \Delta\right)=\frac{1}{2} \rho(\mathbf{0}, \partial \Delta) \geqslant \varepsilon,
$$

т.е. $\Delta$ не лежит в $\varepsilon$-окрестности множества $P_{G}$, а стало быть, не лежит и в $\varepsilon$-окрестности множества $G$, что противоречит условию леммы. Следовательно, $\frac{1}{2} \Delta \subseteq$ $\mathscr{P}_{G}$ и $\operatorname{vol}_{k}\left(\mathscr{P}_{G}\right) \geqslant 2^{-k} \operatorname{vol}_{k} \Delta$.

Стало быть,

$$
\operatorname{vol}_{n-1} G>(2 n)^{-k} \operatorname{vol}_{k} \Delta \cdot \operatorname{vol}_{n-k-1}\left(G \cap L_{2}\right) .
$$


Лемма 20. Пусть $\mathbb{R}^{n}=L_{1} \oplus L_{2}, \operatorname{dim} L_{1}=k \leqslant n-2, \operatorname{dim} L_{2}=n-k$. Пусть угол между $L_{1}$ и $L_{2}$ равен $\alpha$. Пусть $G \subset \mathbb{R}^{n}-(n-1)$-мерное выпуклое компактное множество. Пусть $\Delta \subset L_{1}-k$-мерное выпуклое компактное множсество, содержащее 0 и лежащее в $\varepsilon$-окрестности множества $G$. Пусть

$$
\rho(\mathbf{0}, \partial \Delta) \geqslant \frac{2 n \varepsilon}{\sin \alpha} .
$$

Тогда

$$
\operatorname{vol}_{n-1} G>\frac{(\sin \alpha)^{n-k}}{2^{k} \cdot n^{k+1}} \operatorname{vol}_{k} \Delta \cdot \operatorname{vol}_{n-k-1}\left(G \cap L_{2}\right) .
$$

ДокАЗАТЕЛЬСТво. Обозначим через $L_{2}^{\prime}$ ортогональное дополнение к $L_{1}$ и рассмотрим оператор $A$ из лемм 15 и 16. Поскольку для всех $\mathbf{x}, \mathbf{y} \in \mathbb{R}^{n}$ имеет место неравенство $(23)$, то $\rho(\mathbf{0}, \partial \Delta) \geqslant 2 \varepsilon^{\prime}$ и $\Delta$ лежит в $\varepsilon^{\prime}$-окрестности множества $A(G)$, где $\varepsilon^{\prime}=n \varepsilon / \sin \alpha$. Следовательно, мы можем воспользоваться леммой 19 и получить, что

$$
\operatorname{vol}_{n-1}(A(G)) \geqslant(2 n)^{-k} \operatorname{vol}_{k} \Delta \cdot \operatorname{vol}_{n-k-1}\left(A(G) \cap L_{2}^{\prime}\right) .
$$

С другой стороны, по лемме 15

$$
|A(\mathbf{x})-A(\mathbf{y})|>\frac{\sin \alpha}{n}|\mathbf{x}-\mathbf{y}| .
$$

Следовательно,

$$
\rho(\mathbf{0}, \operatorname{aff}(A(G)))>\frac{\sin \alpha}{n} \rho(\mathbf{0}, \operatorname{aff}(G)),
$$

откуда видим, что

$$
\operatorname{vol}_{n-1}(A(G))<\frac{n}{\sin \alpha} \operatorname{vol}_{n-1} G,
$$

поскольку оператор $A$ не меняет $n$-мерный объем.

По лемме $15|A(\mathbf{x})| \geqslant \sin \alpha|\mathbf{x}|$ для всех $\mathbf{x} \in L_{2}$. Стало быть,

$$
\operatorname{vol}_{n-k-1}\left(A(G) \cap L_{2}^{\prime}\right) \geqslant(\sin \alpha)^{n-k-1} \operatorname{vol}_{n-k-1}\left(G \cap L_{2}\right) .
$$

Подставляя (27) и (28) в (25), получаем

$$
\operatorname{vol}_{n-1} G>\frac{(\sin \alpha)^{n-k}}{2^{k} \cdot n^{k+1}} \operatorname{vol}_{k} \Delta \cdot \operatorname{vol}_{n-k-1}\left(G \cap L_{2}\right) .
$$

Лемма 21. Пусть $P \subset \mathbb{R}^{n}-n$-мерный выпуклый многогранник. Пусть количество $(n-1)$-мерных граней $P$ равно $p$. Тогда найдется такой выпукльий многогранник $Q$, содержащий $P$, что каждая его $(n-1)$-мерная грань $F_{Q}$ содержит некоторую $(n-1)$-мерную грань $F_{P}$ многогранника $P$, удовлетворяющую следующему условию:

$$
\operatorname{vol}_{n-1} F_{P} \cdot \operatorname{diam} P \geqslant \frac{1}{p} \operatorname{vol}_{n} P .
$$


ДоказАТельСтво. Обозначим через $\mathbb{F}$ множество $(n-1)$-мерных граней $P$ объема не меньше

$$
\frac{\operatorname{vol}_{n} P}{p \operatorname{diam} P} .
$$

Это множество непусто. Действительно, если через $\mathbf{n}_{F}$ обозначить нормаль к плоскости $\operatorname{aff}(F)$, направленную в полупространство, содержашее $P$, то получим, что $P \subseteq \bigcup\left\{\mathbf{x}+\lambda \mathbf{n}_{F} \mid \mathbf{x} \in F, 0 \leqslant \lambda \leqslant \operatorname{diam} F\right\}$, где объединение берется по всем граням $P$. Поэтому если $\mathbb{F}=\varnothing$, то объем $P$ обязан быть меньше $\operatorname{vol}_{n} P$. Следовательно, $\mathbb{F} \neq \varnothing$.

Определим теперь полупространства

$$
H(F)=\left\{\mathbf{x}+\lambda \mathbf{n}_{F} \mid \mathbf{x} \in \operatorname{aff}(F), \lambda \geqslant 0\right\}
$$

и множество

$$
Q=\bigcap_{F \in \mathbb{F}} H(F)
$$

и докажем, что $Q$ - искомый многогранник.

Достаточно доказать, что $Q$ ограничено. Допустим противное.

Если для любой грани $F \notin \mathbb{F}$ множество $Q \cap H(F)$ ограничено, то положим $Q^{\prime}=Q, \mathbb{F}^{\prime}=\mathbb{F}$.

Если же это неверно, то проделаем следующую процедуру. Пусть грань $F_{1} \notin \mathbb{F}$ такова, что множество $Q \cap H\left(F_{1}\right)$ не ограничено.Тогда положим $\mathbb{F}_{1}=\mathbb{F} \cup\left\{F_{1}\right\}$, $Q_{1}=Q \cap H\left(F_{1}\right)$. Если же опять сушествует грань $F_{2} \notin \mathbb{F}_{1}$ такая, что множество $Q_{1} \cap H\left(F_{2}\right)$ не ограничено, то положим $\mathbb{F}_{2}=\mathbb{F}_{1} \cup\left\{F_{2}\right\}, Q_{2}=Q_{1} \cap H\left(F_{2}\right)$. И так далее, пока не получим множества $\mathbb{F}_{k}$ и $Q_{k}$ такие, что для любой грани $F \notin \mathbb{F}_{k}$ множество $Q_{k} \cap H(F)$ ограничено. Положим $Q^{\prime}=Q_{k}, \mathbb{F}^{\prime}=\mathbb{F}_{k}$.

Итак, в обоих случаях множество

$$
Q^{\prime}=\bigcap_{F \in \mathbb{F}^{\prime}} H(F)
$$

не ограничено, в то время как для любой грани $F \notin \mathbb{F}^{\prime}$ множество $Q^{\prime} \cap H(F)$ ограничено.

Поскольку $Q^{\prime}$ выпукло и не ограничено, то существует такой вектор $\gamma \in \mathbb{R}^{n}$, что $\forall \mathbf{x} \in Q^{\prime}$ выполняется включение $\{\mathbf{x}+\lambda \boldsymbol{\gamma} \mid \lambda \geqslant 0\} \subset Q^{\prime}$, а стало быть, и

$$
M=\{\mathbf{x}+\lambda \boldsymbol{\gamma} \mid \mathbf{x} \in P, \lambda \geqslant 0\} \subseteq Q^{\prime}
$$

Далее, для любого $\mathbf{x} \in \operatorname{int} P$ существует такое $0 \leqslant \lambda \leqslant \operatorname{diam} P$, что $\mathbf{x}+\lambda \gamma \in \partial P$. При этом $\mathbf{x}+\lambda \boldsymbol{\gamma} \in \operatorname{int} M$ и, стало быть, $\mathbf{x}+\lambda \boldsymbol{\gamma} \in \partial P \backslash \partial M$, откуда делаем вывод, что

$$
P \subseteq M^{-}=\{\mathbf{x}-\lambda \boldsymbol{\gamma} \mid \mathbf{x} \in \partial P \backslash \partial M, 0 \leqslant \lambda \leqslant \operatorname{diam} P\} .
$$

Следовательно,

$$
\operatorname{diam} P \cdot \operatorname{vol}_{n-1}(\partial P \backslash \partial M) \geqslant \operatorname{vol}_{n} M^{-} \geqslant \operatorname{vol}_{n} P .
$$


Но $\partial P \backslash \partial M$ состоит из менее чем $p$ граней $P$, значит, в $\partial P \backslash \partial M$ должна содержаться грань объема больше чем

$$
\frac{\operatorname{vol}_{n} P}{p \operatorname{diam} P}
$$

Но ни одна из граней $P$, лежащих в $\partial P \backslash \partial M$, не принадлежит $\mathbb{F}^{\prime}$, поскольку $M \subseteq$ $Q^{\prime}$. Получаем противоречие, доказьвающее, что множество $Q$ ограничено и, стало быть, является искомым многогранником.

Лемма 22. Пусть $S_{o} \subset \mathbb{R}^{n}$ - стандартный $(n-1)$-мерный симплекс. Пусть $\pi$ - произвольная гиперплоскость, проходящая через $\mathbf{0}$. Пусть $\mathbf{a} \in S_{o} \backslash \pi$, $\left|\mathbf{a}-c\left(S_{o}\right)\right| \leqslant 1 /(2 n)$. Множество вершин $S_{o}$, находящихся в одном замкнутом полупространстве (относительно $\pi$ ) с точкой $\mathbf{a}$, обозначим $V_{1}$. Множество оставшихся вериин обозначим $V_{2}$. Тогда в множестве $V_{1}$ найдется такая точка $\mathbf{v}$, что

$$
\rho\left(\mathbf{v}, \pi \cap \operatorname{aff}\left(S_{o}\right)\right)>\frac{1}{2 n} .
$$

ДокАЗАТЕльСтво. Рассмотрим гиперплоскость $\pi^{\prime}$, проходяшую через 0 и $c\left(S_{o}\right)$ и такую, что $\pi \cap \operatorname{aff}\left(S_{o}\right)$ параллельно $\pi^{\prime} \cap \operatorname{aff}\left(S_{o}\right)$. Рассмотрим нормированную линейную форму $\mathscr{L}(\mathbf{x})$, равную нулю на $\pi^{\prime}$ и неотрицательную на $V_{1}$. Поскольку $c\left(S_{o}\right) \in \pi^{\prime}$ и симплекс $S_{o}$ правильньй, то в $S_{o}$ найдется точка $\mathbf{x}$, для которой $\mathscr{L}(\mathbf{x})=R\left(S_{o}\right)$. Следовательно, в $V_{1}$ найдется такая точка $\mathbf{v}$, что $\mathscr{L}(\mathbf{v}) \geqslant R\left(S_{o}\right)$. Стало быть,

$$
\rho\left(\mathbf{v}, \pi^{\prime}\right)=\mathscr{L}(\mathbf{v}) \geqslant R\left(S_{o}\right)=\frac{1}{\sqrt{n(n-1)}}>\frac{1}{n} .
$$

Если $\pi^{\prime}$ отделяет а от $\pi \cap \operatorname{aff}\left(S_{o}\right)$ или же если а находится строго между $\pi \cap$ $\operatorname{aff}\left(S_{o}\right)$ и $\pi^{\prime} \cap \operatorname{aff}\left(S_{o}\right)$, то доказывать нечего, ибо тогда $\rho\left(\mathbf{v}, \pi \cap \operatorname{aff}\left(S_{o}\right)\right)$ будет заведомо больше $1 / n$. Остается рассмотреть лиш случай, когда $\pi$ отделяет а от $\pi^{\prime} \cap \operatorname{aff}\left(S_{o}\right)$. Но тогда расстояние между $\pi \cap \operatorname{aff}\left(S_{o}\right)$ и $\pi^{\prime} \cap \operatorname{aff}\left(S_{o}\right)$ не превосходит $1 /(2 n)$. Стало быть, в этом случае $\rho\left(\mathbf{v}, \pi \cap \operatorname{aff}\left(S_{o}\right)\right)>1 /(2 n)$. Лемма доказана.

Теперь мы можем приступить к непосредственному доказательству теоремы 3.

ДоКАЗАТЕЛЬСТВО ТЕОРЕМЫ 3. Итак, мы предполагаем, что определителиграней паруса П равномерно ограничены константой $D$.

Положим

$$
\lambda=\left(8 n^{3} \cdot 8^{(n D)^{n}}\right)^{-(n+1)}
$$

и допустим, что в парусе П сушествует грань $F$ такая, что

$$
\frac{\operatorname{det} F}{\operatorname{det} S(F)} \leqslant B_{n-1} \frac{(n-1) !}{\sqrt{n}}\left(\frac{1}{3 n(n-1)}\left(\frac{\lambda}{2}\right)^{2^{n-1}}\right)^{n-1} .
$$

Предположив сушествование такой грани, мы докажем, что определитель одной из соседних с $F$ граней больше $D$, откуда будет следовать утверждение теоремы. 
Применив подходящий гиперболический поворот (который, очевидно, не изменит ни комбинаторной структуры паруса, ни определителей его граней), мы можем, не ограничивая общности, считать, что $F$ перпендикулярно биссектрисе конуса $\mathscr{C}_{o}$.

Перейдем теперь от решетки $\Lambda$ к решетке

$$
\Lambda^{\prime}=(\operatorname{det} S(F))^{-1 / n} \Lambda \text {. }
$$

Полиэдр $K$ перейдет в полиэдр $K^{\prime}$, парус П перейдет в парус $\Pi^{\prime}$, грань $F$ паруса П перейдет в грань $F^{\prime}$ паруса $\Pi^{\prime}$. Определители граней паруса П' будут ограничены константой $D \cdot(\operatorname{det} S(F))^{-1}$. Очевидно, $S\left(F^{\prime}\right)$ совпадает со стандартным $(n-1)$ мерньп симплексом, который мы, как и прежде, обозначаем $S_{o}$.

$$
\begin{aligned}
& \text { Поскольку vol }_{n-1} S_{o}=\frac{\sqrt{n}}{(n-1) !} \text {, то } \\
& \operatorname{vol}_{n-1} F^{\prime}<B_{n-1}\left(\frac{1}{3 n(n-1)}\left(\frac{\lambda}{2}\right)^{2^{n-1}}\right)^{n-1} .
\end{aligned}
$$

Наша задача теперь сводится к доказательству того, что определитель одной из соседних с $F^{\prime}$ граней паруса $\Pi^{\prime}$ больше $D \operatorname{det} \Lambda^{\prime}$.

Изложим схему доказательства. В трехмерном случае мы пользовались тем, что вершины $F^{\prime}$ находятся в окрестности некоторых вершин $S_{o}$. За счет этого мы могли выбрать $(n-2)$-мерную грань $G$ многогранника $F^{\prime}$, ближайшую к $c\left(S_{o}\right)$, по которой граничит с $F^{\prime}$ искомая грань паруса. В $n$-мерном случае такой близости вершин может не быть - может оказаться, что $F^{\prime}$ даже содержит $c\left(S_{o}\right)$. Поэтому мы будем действовать следующим образом. Сначала мы приблизим $F^{\prime}$ многогранником $\Gamma$ меньшей размерности, $\operatorname{dim} \Gamma=k \leqslant n-2$. Затем мы построим $(n-k-1)$-мерное сечение $P$ многогранника $F^{\prime}$ плоскостью $\pi_{n-k-1}$, содержащейся в $\operatorname{aff}\left(S_{o}\right)$ и проходящей через точки $\mathbf{b}$ и $c(\Delta)$, где $\mathbf{b}-$ точка, близкая к $c\left(S_{o}\right)$ и при этом не очень близкая к $\Gamma$, а $\Delta$ - достаточно большой $k$-мерный симплекс, лежащий в $Г$.

Пользуясь леммой 16 , получаем, что $\operatorname{vol}_{n-k-1}(P) \cdot \operatorname{vol}_{k}(\Gamma)$ достаточно велико по сравнению $\mathrm{c} \mathrm{vol}_{n-1}\left(F^{\prime}\right)$. Далее приходится рассматривать два случая: $k=$ $n-2$ и $k<n-2$. За счет выбора симплекса $\Delta$ достаточно большим в случае $k=n-2$, пользуясь леммой 17 , получаем, что объем каждой $(n-2)$-мерной грани многогранника $F^{\prime}$, пересекаюшейся с $P$, достаточно велик по сравнению с $\operatorname{vol}_{k}(\Delta)$ и $\operatorname{vol}_{k}(\Gamma)$. В случае $k<n-2$ мы пользуемся леммой 20 и получаем, что объем каж дой $(n-2)$-мерной грани $G$ многогранника $F^{\prime}$, пересекающейся с $P$, достаточно велик по сравнению $\operatorname{col}_{k}(\Gamma) \cdot \operatorname{vol}_{n-k-2}(P \cap G)$.

Пользуясь этими соотношениями, в каждом из указанных двух случаев среди $(n-2)$-мерных граней многогранника $F^{\prime}$, пересекающихся с $P$, выбирается такая грань $G_{o}$, что, во-первых, $F^{\prime}$ и точка $\mathbf{b}$ лежат по разные стороны от плоскости $\operatorname{aff}\left(G_{o}\right)$ (при этом точку b в случае $k<n-2$ приходится немного шевелить) и, во-вторых, $\operatorname{vol}_{n-2}\left(G_{o}\right) \cdot \operatorname{diam} P$ достаточно велико по сравнению $\operatorname{col}_{n-1}\left(F^{\prime}\right)$.

Затем мы рассматриваем грань $F^{\prime \prime}$ паруса $\Pi^{\prime}$, граничащую с $F^{\prime}$ по $G_{o}$. Пользуясь тем, что целочисленное (относительно $\Lambda^{\prime}$ ) расстояние от $F^{\prime}$ до $\mathbf{0}$ не больше $D$, получаем, что все вершины $F^{\prime \prime}$, не лежащие в $G_{o}$, находятся довольно далеко от $G_{o}$. Остается заметить, что $\operatorname{diam} P$ довольно мал, $\operatorname{a~vol}_{n-2}\left(G_{o}\right) \cdot \operatorname{diam} P$ достаточно велико по сравнению с $\operatorname{vol}_{n-1}\left(F^{\prime}\right)$, и мы сразу получим, что $\operatorname{vol}_{n-1}\left(F^{\prime \prime}\right)$ 
слишком велик по сравнению $\mathrm{c} \operatorname{vol}_{n-1}\left(F^{\prime}\right)$. Отсюда уже несложно будет вывести, что $\operatorname{det} F^{\prime \prime} \geqslant D \operatorname{det} \Lambda^{\prime}$.

На этом мы завершаем изложение схемы и приступаем к подробному доказательству того, что определитель одной из соседних с $F^{\prime}$ граней паруса П' больше $D \operatorname{det} \Lambda^{\prime}$.

Применив лемму 6 , получим, что $\operatorname{diam} F^{\prime}>1 /(3 n(n-1))$. Стало быть,

$$
\operatorname{vol}_{n-1} F^{\prime}<B_{n-1}\left(\operatorname{diam} F^{\prime} \cdot\left(\frac{\lambda}{2}\right)^{2^{n-1}}\right)^{n-1} .
$$

Обозначим вершины $F^{\prime}$ через $\mathbf{v}_{1}, \ldots, \mathbf{v}_{m}$. По лемме 7 найдутся точки $\boldsymbol{\zeta}_{1}, \ldots, \boldsymbol{\zeta}_{m}$, лежащие в плоскости $\operatorname{aff}\left(F^{\prime}\right)=\left\{x_{1}+\cdots+x_{n}=1\right\}$, такие, что

$$
\max _{1 \leqslant i \leqslant m}\left|\mathbf{v}_{i}-\boldsymbol{\zeta}_{i}\right| \leqslant \lambda R(\Gamma),
$$

где $\Gamma=\operatorname{conv}\left(\boldsymbol{\zeta}_{1}, \ldots, \boldsymbol{\zeta}_{m}\right)$, причем $\operatorname{dim} \Gamma=k \leqslant n-2$. Обозначим

$$
\varepsilon=\max _{1 \leqslant i \leqslant m}\left|\mathbf{v}_{i}-\zeta_{i}\right|
$$

и убедимся, что $\varepsilon<\lambda$. Действительно,

$$
R(\Gamma) \leqslant \frac{1}{2} \operatorname{diam} \Gamma \leqslant \frac{1}{2}\left(\operatorname{diam} F^{\prime}+2 \varepsilon\right) \leqslant \frac{1}{\sqrt{2}}+\lambda R(\Gamma)
$$

откуда $R(\Gamma) \leqslant 1 /(\sqrt{2}(1-\lambda))<1$, поскольку $\lambda<1 / 4$. Следовательно, $\varepsilon<\lambda$.

Поскольку $\operatorname{det} F^{\prime} \leqslant D \operatorname{det} \Lambda^{\prime}$, то $F^{\prime}$ разбивается на не более чем $D$ пустых целочисленных (относительно $\Lambda^{\prime}$ ) симплексов. Отсюда видим, что число вершин $F^{\prime}$ не превосходит $n D$. То есть $m \leqslant n D$. Воспользовавшись леммой 9 , мы можем найти симплекс $\Delta \subseteq$ Г объема

$$
\operatorname{vol}_{k} \Delta \geqslant 8^{-(n D)^{n}} \operatorname{vol}_{k} \Gamma=8 n^{3} \lambda^{1 /(n+1)} \operatorname{vol}_{k} \Gamma
$$

и при этом такой, что любой симплекс с вершинами из множества $\left\{\boldsymbol{\zeta}_{1}, \ldots, \boldsymbol{\zeta}_{m}\right\}$ либо не пересекается с int $\Delta$, либо содержит $\Delta$. Из леммы 10 получаем, что

$$
R(\Delta) \geqslant R(\Gamma) 8 n^{3} \lambda^{1 /(n+1)}>8 n^{3} \varepsilon .
$$

По лемме 11 отсюда следует, что

$$
\rho(c(\Delta), \partial \Delta)>16 n^{2} \varepsilon .
$$

Возьмем точку $\mathbf{b} \in S_{o}$ такую, что $\left|\mathbf{b}-c\left(S_{o}\right)\right|=1 /(4 n)$ и $\rho(\mathbf{b}, \operatorname{aff}(\Gamma)) \geqslant 1 /(4 n)$. Плоскость размерности $n-k-2$, содержащуюся в $\left\{x_{1}+\cdots+x_{n}=1\right\}$, проходящую через $c(\Delta)$ и ортогональную плоскости $\mathrm{aff}(\Gamma \cup\{\mathbf{b}\})$, обозначим $\pi_{n-k-2}$. Отметим, что когда $\operatorname{aff}(\Gamma \cup\{\mathbf{b}\})=\left\{x_{1}+\cdots+x_{n}=1\right\}$ (т.е. в случае $k=n-2$ ), плоскость $\pi_{n-k-2}$ вырождается в точку $c(\Delta)$. 
Положим

$$
\pi_{n-k-1}=\operatorname{aff}\left(\pi_{n-k-2} \cup\{\mathbf{b}\}\right) \quad \text { и } P=F^{\prime} \cap \pi_{n-k-1} .
$$

Легко видеть, что

$$
b \notin P,
$$

поскольку $P$ как подмножество $F^{\prime}$ лежит в $\varepsilon$-окрестности $\Gamma$, в то время как $\varepsilon<\lambda<$ $1 /(4 n) \leqslant \rho(\mathbf{b}, \operatorname{aff}(\Gamma))$.

Угол между прямой aff $(\mathbf{b}, c(\Delta))$ и плоскостью aff $(\Gamma)$ (он же угол между $\pi_{n-k-1}$ и $\operatorname{aff}(\Gamma))$ обозначим $\alpha$. Поскольку $|\mathbf{b}-c(\Delta)|<\varepsilon+\operatorname{diam} S_{o}<2$, то

$$
\sin \alpha>\frac{1}{8 n}
$$

откуда $2(n-1) / \sin \alpha<16 n^{2}$. Следовательно, из (30) получаем

$$
\rho(c(\Delta), \partial \Gamma) \geqslant \rho(c(\Delta), \partial \Delta)>\frac{2(n-1)}{\sin \alpha} \varepsilon
$$

С другой стороны, $\operatorname{vol}_{k} \Delta \geqslant 8 n^{3} \lambda^{1 /(n+1)} \operatorname{vol}_{k} \Gamma$, откуда по лемме 12

$$
\frac{\left|\mathbf{a}_{1}-c(\Delta)\right|}{\left|\mathbf{a}_{2}-c(\Delta)\right|} \geqslant \frac{8 n^{3}}{k(k+1)} \lambda^{1 /(n+1)}>8 \lambda^{1 /(n+1)}
$$

для любых двух точек $\mathbf{a}_{1}, \mathbf{a}_{2} \in \partial \Gamma$, лежащих на одной прямой с $c(\Delta)$.

Итак, множества $F^{\prime}$ и $Г$ удовлетворяют всем требованиям леммы 16 (в качестве начала координат нужно взять точку $c(\Delta)$, а в качестве объемлюшего пространства - плоскость $\left.\left\{x_{1}+\cdots+x_{n}=1\right\}\right)$. Применяя эту лемму, получаем

$$
\operatorname{vol}_{n-k-1}(P) \cdot \operatorname{vol}_{k}(\Gamma)>\lambda^{(n-1) /(n+1)} \operatorname{vol}_{n-1}\left(F^{\prime}\right)
$$

В частности, отсюда следует, что $P$ - это $(n-k-1)$-мерный многогранник (очевидно, выпуклый). Граница $P$ является пересечением плоскости $\pi_{n-k-1}$ с объединением каких-то из $(n-2)$-мерных граней многогранника $F^{\prime}$. Множество всех $(n-2)$-мерных граней $F^{\prime}$, имеющих непустое пересечение с $P$, но не содержащих $P$, обозначим $\mathbb{G}$.

Докажем, что $\Delta$ лежит в $\varepsilon$-окрестности любой грани из множества $\mathbb{G}$. Пусть $G \in \mathbb{G}$, и пусть $\mathbf{v}_{i_{1}}, \ldots, \mathbf{v}_{i_{\nu}}-$ вершины $G$. Тогда для каждого $\eta=1, \ldots, \nu$ выполнено неравенство $\left|\mathbf{v}_{i_{\eta}}-\boldsymbol{\zeta}_{i_{\eta}}\right| \leqslant \varepsilon$, т.е.

$$
\rho_{H}\left(G, \operatorname{conv}\left(\zeta_{i_{1}}, \ldots, \zeta_{i_{\nu}}\right)\right) \leqslant \varepsilon
$$

С другой стороны, $P$ содержится в (открытой) окрестности радиуса $8 n \varepsilon$ точки $c(\Delta)$, поскольку $F^{\prime}$ находится в $\varepsilon$-окрестности $\Gamma$, а $\sin \alpha>1 /(8 n)$. Следовательно,

$$
\rho(G, c(\Delta))<8 n \varepsilon
$$

так как $G \cap P \neq \varnothing$. 
Из (30), (35) и (36) получаем

$$
\rho\left(c(\Delta), \operatorname{conv}\left(\boldsymbol{\zeta}_{i_{1}}, \ldots, \boldsymbol{\zeta}_{i_{\nu}}\right)\right)<(1+8 n) \varepsilon<16 n^{2} \varepsilon<\rho(c(\Delta), \partial \Delta),
$$

т.е. int $\Delta$ имеет непустое пересечение с $\operatorname{conv}\left(\boldsymbol{\zeta}_{i_{1}}, \ldots, \boldsymbol{\zeta}_{i_{\nu}}\right)$. Но симплекс $\Delta$ мы выбирали согласно лемме 9, стало быть, $\Delta \subseteq \operatorname{conv}\left(\boldsymbol{\zeta}_{i_{1}}, \ldots, \zeta_{i_{\nu}}\right)$. Из неравенства $(35)$ теперь следует, что, действительно, $\Delta$ содержится в $\varepsilon$-окрестности $G$.

Учитьвая (33), получаем, что множества $G$ и $\Delta$ в случае $k=n-2$ удовлетворяют лемме 17 , а в случае $k<n-2$ удовлетворяют лемме 20 (как и прежде, в качестве начала координат нужно взять точку $c(\Delta)$, а в качестве объемлющего пространства - плоскость $\left.\left\{x_{1}+\cdots+x_{n}=1\right\}\right)$. Если $k=n-2$, то из леммы 17 и соотношения (29) следует, что

$$
\operatorname{vol}_{n-2}(G) \geqslant 2^{-(n-2)} \operatorname{vol}_{k}(\Delta)>16 n \cdot 2^{-n} \lambda^{1 /(n+1)} \operatorname{vol}_{k}(\Gamma),
$$

а если $k<n-2$, то из леммы 20 и соотношений $(29),(32)$ следует, что

$$
\begin{aligned}
\operatorname{vol}_{n-2}(G) & \geqslant \frac{(\sin \alpha)^{n-k-1}}{2^{k}(n-1)^{k+1}} \operatorname{vol}_{k}(\Delta) \cdot \operatorname{vol}_{n-k-2}(P \cap G) \\
& >16 n(4 n)^{-2 n} \lambda^{1 /(n+1)} \operatorname{vol}_{k}(\Gamma) \cdot \operatorname{vol}_{n-k-2}(P \cap G) .
\end{aligned}
$$

Вспомним, что $P$ содержится в открытой окрестности радиуса $8 n \varepsilon$ точки $c(\Delta)$. Отсюда видим, что

$$
\operatorname{diam} P<16 n \varepsilon
$$

Если $k=n-2$, то $\pi_{n-k-1}=\operatorname{aff}(\mathbf{b}, c(\Delta))$, а $P$ - некоторый отрезок этой прямой, не содержащий точку $\mathbf{b}$ (см. (31)). Обозначим через $G_{o}$ такую грань из $\mathbb{G}$, что гиперплоскость $\operatorname{aff}\left(G_{o} \cup\{\mathbf{0}\}\right)$ отделяет $\mathbf{b}$ от $P$ (а стало быть, и от $\left.F^{\prime}\right)$. Применим к $G_{o}$ неравенство (37), домноженное на $\varepsilon$, и подставим в него последовательно (39) и $(34)$ :

$$
\begin{aligned}
& \operatorname{vol}_{n-2}\left(G_{o}\right) \cdot \varepsilon>2^{-n} \lambda^{1 /(n+1)} \operatorname{vol}_{n-2}(\Gamma) \cdot \operatorname{diam}(P) \\
& \quad=2^{-n} \lambda^{1 /(n+1)} \operatorname{vol}_{n-2}(\Gamma) \cdot \operatorname{vol}_{1}(P)>2^{-n} \lambda^{n /(n+1)} \operatorname{vol}_{n-1}\left(F^{\prime}\right)
\end{aligned}
$$

Если же $k<n-2$, то мы можем применить к многограннику $P$ лемму 21 . Количество $(n-k-2)$-мерных граней $P$ не превьшшает количества $(n-2)$-мерных граней многогранника $F^{\prime}$, которое, в свою очередь, не больше чем $n D$ в силу того, что $F^{\prime}$ можно разбить на $D$ симплексов, у каждого из которых ровно $n$ гиперграней. Следовательно, применив лемму 21 , мы получим многогранник $Q \subset \pi_{n-k-1}$, содержаший $P$, такой, что каждая его $(n-k-2)$-мерная грань $G_{Q}$ содержит некоторую $(n-k-2)$-мерную грань $G_{P}$ многогранника $P$, удовлетворяюшую следуюшему условию:

$$
\operatorname{vol}_{n-k-2}\left(G_{P}\right) \cdot \operatorname{diam}(P) \geqslant \frac{1}{n D} \operatorname{vol}_{n-k-1}(P) .
$$

Открытый шар радиуса $8 n \varepsilon$ с центром в $c(\Delta)$ содержит $P$ и, стало быть, пересекается с плоскостями всех $(n-k-2)$-мерных граней многогранника $Q$. Следовательно, $R(Q)<8 n \varepsilon$. Рассмотрим $(n-k-1)$-мерный шар радиуса $8 n \varepsilon$ с центром 
в $\mathbf{b}$, лежаший в плоскости $\pi_{n-k-1}$. Поскольку $R(Q)<8 n \varepsilon$, то этот шар не содержится в $Q$. Стало быть, в плоскости $\pi_{n-k-1}$ найдется точка $\mathbf{a}$, не лежащая в $Q$ и такая, что $|\mathbf{a}-\mathbf{b}|=8 n \varepsilon$. Отметим, что

$$
\left|\mathbf{a}-c\left(S_{o}\right)\right| \leqslant \frac{1}{4 n}+8 n \varepsilon<\frac{1}{2 n},
$$

так как $\varepsilon<\lambda<\left(32 n^{2}\right)^{-1}$.

Многогранник $Q$ не содержит точку а. Значит, найдется $(n-k-2)$-мерная плоскость, лежашая в $\pi_{n-k-1}$, содержашая некоторую $(n-k-2)$-мерную грань многогранника $Q$ (и, стало быть, некоторую $(n-k-2)$-мерную грань многогранника $P$ ) и такая, что а и $Q$ лежат по разные стороны от нее.

Следовательно, существует грань $G_{o} \in \mathbb{G}$ такая, что, во-первых, гиперплоскость $\operatorname{aff}\left(G_{o} \cup\{\boldsymbol{0}\}\right)$ отделяет а от $F^{\prime}$, а во-вторых, учитывая (41),

$$
\operatorname{vol}_{n-k-2}\left(P \cap G_{o}\right) \cdot \operatorname{diam}(P) \geqslant \frac{1}{n D} \operatorname{vol}_{n-k-1}(P) .
$$

Применим к $G_{o}$ неравенство $(38)$, домноженное на $\varepsilon$, и подставим в него последовательно (39), (42) и (34):

$$
\begin{aligned}
\operatorname{vol}_{n-2}\left(G_{o}\right) \cdot \varepsilon & >(4 n)^{-2 n} \lambda^{1 /(n+1)} \operatorname{vol}_{k}(\Gamma) \cdot \operatorname{vol}_{n-k-2}\left(P \cap G_{o}\right) \cdot \operatorname{diam}(P) \\
& \geqslant(n D)^{-1}(4 n)^{-2 n} \lambda^{1 /(n+1)} \operatorname{vol}_{k}(\Gamma) \cdot \operatorname{vol}_{n-k-1}(P) \\
& >(n D)^{-1}(4 n)^{-2 n} \lambda^{n /(n+1)} \operatorname{vol}_{n-1}\left(F^{\prime}\right) .
\end{aligned}
$$

Итак, при $k=n-2$ мы получили неравенство (40), а при $k<n-2$ - неравенство (43). В (43) константа хуже, чем в (40), поэтому можно считать, что при любом значении $k$ мы нашли грань $G_{o}$ многогранника $F^{\prime}$ и точку $\mathbf{a} \in S_{o}$ (в случае $k=n-2$ можно положить $\mathbf{a}=\mathbf{b})$ такие, что $\left|\mathbf{a}-c\left(S_{o}\right)\right|<1 /(2 n)$,

$$
\operatorname{vol}_{n-2}\left(G_{o}\right) \cdot \varepsilon>\frac{\lambda^{n /(n+1)}}{n D(4 n)^{2 n}} \operatorname{vol}_{n-1}\left(F^{\prime}\right),
$$

и такие, что а и $F^{\prime}$ лежат по разные стороны от $\operatorname{aff}\left(G_{o}\right)$ в плоскости $\left\{x_{1}+\cdots+\right.$ $\left.x_{n}=1\right\}$.

Положим $\pi=\operatorname{aff}\left(G_{o} \cup\{\boldsymbol{0}\}\right)$. Множество вершин $S_{o}$, находящихся в одном замкнутом полупространстве с точкой а (относительно $\pi$ ), обозначим $V_{1}$. Множество оставшихся вершин $S_{o}$ обозначим $V_{2}$. По лемме 22 в $V_{1}$ найдется такая точка $\mathbf{v}$, что $\rho\left(\mathbf{v}, \pi \cap \operatorname{aff}\left(S_{o}\right)\right)>1 /(2 n)$. Отсюда видим, что для любой точки $\mathbf{w} \in V_{2}$

$$
\rho(\mathbf{v}, \pi \cap[\mathbf{v}, \mathbf{w}])>\frac{1}{2 n} .
$$

Рассмотрим грань $F^{\prime \prime}$ паруса $\Pi^{\prime}$, граничашую с $F^{\prime}$ по $G_{o}$. Плоскость aff $\left(F^{\prime \prime}\right)$ является опорной для полиэдра $K^{\prime}$ и при этом она отделяет $\mathbf{0}$ от $K^{\prime}$. С другой стороны, $\operatorname{aff}\left(F^{\prime \prime}\right)$ отделяет $V_{1}$ от $K^{\prime}$, поскольку $V_{1}$ и $F^{\prime}$ лежат по разные стороны от $\operatorname{aff}\left(G_{o}\right)$ в плоскости $\left\{x_{1}+\cdots+x_{n}=1\right\}$, в то время как $\operatorname{aff}\left(G_{o}\right) \subset \operatorname{aff}\left(F^{\prime \prime}\right)$. Стало быть, $\mathbf{0}$ и $V_{1}$ лежат по одну сторону относительно плоскости $\operatorname{aff}\left(F^{\prime \prime}\right)$. Но из (45) 
следует, что для любой точки $\mathbf{w} \in V_{2} \rho\left(\mathbf{0}, S\left(F^{\prime \prime}\right) \cap[\mathbf{0}, \mathbf{w}]\right)>(2 n \sqrt{2})^{-1}$. Таким образом, плоскость aff $\left(F^{\prime \prime}\right)$ отсекает от конуса $\mathscr{C}_{o} n$-мерный симплекс, длины ребер которого больше чем $(2 n \sqrt{2})^{-1}$. Отсюда легко видеть, что

$$
\rho\left(\mathbf{0}, \operatorname{aff}\left(F^{\prime \prime}\right)\right)>\frac{1}{2 n \sqrt{2 n}}
$$

Однако имеет место следуюшее соотношение:

$$
\operatorname{vol}_{n-1}\left(F^{\prime \prime}\right) \geqslant \frac{1}{D(n-1) \sqrt{n}} \operatorname{vol}_{n-2}\left(G_{o}\right)
$$

Действительно, поскольку целочисленное (относительно $\Lambda^{\prime}$ ) расстояние от $F^{\prime}$ до $\mathbf{0}$ не больше $D$ (иначе бы $\operatorname{det} F^{\prime}$ был больше, чем $D \operatorname{det} \Lambda^{\prime}$ ), то для любой точки $\mathbf{x} \in \Lambda^{\prime} \backslash \operatorname{aff}\left(F^{\prime}\right)$

$$
\rho\left(\mathbf{x}, \operatorname{aff}\left(F^{\prime}\right)\right) \geqslant \frac{1}{D \sqrt{n}} .
$$

Следовательно, объем $(n-1)$-мерной пирамиды с основанием $G_{o}$ и вершиной из множества $\Lambda^{\prime} \backslash \operatorname{aff}\left(F^{\prime}\right)$ (а такая пирамида обязана содержаться в $F^{\prime \prime}$ ) не меньше чем

$$
\frac{1}{n-1} \cdot \frac{1}{D \sqrt{n}} \operatorname{vol}_{n-2}\left(G_{o}\right)
$$

откуда и получаем (47).

Из неравенств (46), (47) получаем

$$
\operatorname{det} F^{\prime \prime}=(n-1) ! \operatorname{vol}_{n-1}\left(F^{\prime \prime}\right) \cdot \rho\left(\mathbf{0}, \operatorname{aff}\left(F^{\prime \prime}\right)\right)>\frac{(n-2) !}{2 \sqrt{2} D n^{2}} \operatorname{vol}_{n-2}\left(G_{o}\right)
$$

Нетрудно проверить, что $\lambda^{-1 /(n+1)}>4 n^{3} \sqrt{n} D^{3}(4 n)^{2 n}>2 \sqrt{2 n}(n-1) n^{2} D^{3} \times$ $(4 n)^{2 n}$. Следовательно, учитывая (44) и то, что $\varepsilon<\lambda$, из (48) получаем

$$
\begin{aligned}
\operatorname{det} F^{\prime \prime} & >\frac{(n-2) ! \lambda^{-1 /(n+1)}}{2 \sqrt{2} D^{2} n^{3}(4 n)^{2 n}} \operatorname{vol}_{n-1}\left(F^{\prime}\right)>D(n-1) ! \frac{1}{\sqrt{n}} \operatorname{vol}_{n-1}\left(F^{\prime}\right) \\
& =D \operatorname{det} F^{\prime} \geqslant D \operatorname{det} \Lambda^{\prime}
\end{aligned}
$$

что противоречит тому, что определители граней паруса $\Pi^{\prime}$ ограничены константой $D \operatorname{det} \Lambda^{\prime}$. Стало быть, теорема доказана.

В заключение автор хотел бы выразить благодарность Н.Г. Мошевитину за неустанный интерес к настоящей работе и многочисленные обсуждения результатов. 


\section{Список литературы}

1. Erdös P., Gruber M., Hammer J. Latticepoints. Harlow: Longman Scientific \& Technical, 1989. (Pitman Monogr. Surveys Pure Appl. Math. V. 39.)

2. Arnold V. I. A-graded algebras and continued fractions // Comm. Pure Appl. Math. 1989. V. 42. P. $993-1000$.

3. Arnold V. I. Higher dimensional continued fractions // Regul. Chaotic Dyn. 1998. V. 3. №3. P. $10-17$.

4. Tsuchihashi H. Higher dimensional analogues of periodic continued fractions and cusp singularities // Tohoku Math. J. (2). 1983. V. 35. P. 607-639.

5. Lachaud G. Sails and Klein polyhedra // Contemp. Math. 1998. V. 210. P. 373-385.

6. Lachaud G. Voiles et polyèdres de Klein. I, II // Preprints № 95-22, Laboratoire de Mathématiques Discrétes. Marseille-Luminy: C.N.R.S., 1995.

7. Korkina $E$. Classification of $A$-graded algebras with 3 generators // Indag. Math. (N.S.). 1992. V. 3. № 1. P. 27-40.

8. Korkina E. La périodecité des fractions continues multidimensionnelles // C. R. Acad. Sci. Paris Sér. I Math. 1994. V. 319. P. 777-780.

9. Коркина Е. И. Двумерные цепные дроби. Самые простые примеры // Труды МИАН. 1995. Т. 209. С. $143-166$.

10. Брюно А.Д., Парусников В.И. Многогранники Клейна для двух кубических форм Давенпорта // Матем. заметки. 1994. Т. 56. № 4. С. 9-27.

11. Муссафир ЖК.-О. Паруса и базисы Гильберта // Функц. анализ и его прилож. 2000. T. 34. № 2. C. 43-49.

12. Герман О. Н. Паруса и базисы Гильберта // Труды МИАН. 2002. Т. 239. С. 98-105.

13. Kontsevich M. L., Suhov Yu. M. Statistics of Klein polyhedra and multidimensional continued fractions // Amer. Math. Soc. Transl. Ser. 2. 1999. V. 197. P. 9-27.

14. Moussafir J.-O. Convex hulls of integral points // Записки научн. сем. ПОМИ. 2000. T. 256. C. $188-217$.

15. Cassels J. W.S., Swinnerton-Dyer H.P.F. On the product of three homogeneous linear forms and indefinite ternary quadratic forms // Philos. Trans. Roy. Soc. London Ser. A. 1955. V. 248. P. 73-96.

16. Скубенко Б. Ф. Минимумы разложимой кубической формы от трех переменных // Записки научн. сем. ЛОМИ. 1988. Т. 168. Аналитическая теория чисел и теория функций. 9. C. $125-139$.

17. Скубенко Б. Ф. Минимумы разложимых форм степени $n$ от $n$ переменных при $n \geqslant 3 / /$ Записки научн. сем. ЛОМИ. 1990. Т. 183. Модулярные функции и квадратичные формы. 1. C. $142-154$.

18. Боревич З. И., Шафаревич И. Р. Теория чисел. М.: Наука, 1964.

19. Данцер Л., Грюнбаум Б., Кли В. Теорема Хелли. М.: Мир, 1968.

Московский государственный университет

Поступила в редакцию им. М.В. Ломоносова

16.12.2003 и 15.10 .2004 